\title{
The expanded model of cultural intelligence and its explanatory power in the context of expatriation intention
}

\author{
Nicole Franziska Richter* \\ Department of Marketing and Management, \\ University of Southern Denmark (SDU), \\ Campusvej 55, 5230 Odense M, Denmark \\ Email: nicole@sam.sdu.dk \\ *Corresponding author

\section{Christopher Schlaegel} \\ Faculty of Economics and Business, \\ Global Economics \& Management Department, \\ University of Groningen, \\ Nettelbosje 2, 9747 AE Groningen, The Netherlands \\ Email: c.schlagel@rug.nl
}

\author{
Marian van Bakel \\ Department of Marketing and Management, \\ University of Southern Denmark, \\ Sdr. Stationsvej 28, 4200 Slagelse, Denmark \\ Email:msvb@sdu.dk
}

\author{
Robert L. Engle \\ Lender School of Business, \\ Quinnipiac University, \\ 275 Mt Carmel Ave., Hamden, CT, USA \\ Email: robert.engle@quinnipiac.edu
}

\begin{abstract}
The main objective of this study is to test the explanatory power of the expanded cultural intelligence (CQ) scale and its 11 sub-dimensions in predicting expatriation intention (EI) in comparison to the four primary CQ dimensions. We exemplarily outline the theoretical advancement that is possible when focusing on the associations between the CQ sub-dimensions with EI. Based on samples of business students from Germany, the USA and China and using regression analysis and necessary condition analysis, our results indicate that the CQ dimensions are relevant determinants of EI, explaining variance over and above established determinants. Moreover, the results show that the CQ sub-dimensions explain more variance in EI than the four primary dimensions and offer potential to advance theorising in the field.
\end{abstract}


Keywords: cultural intelligence; CQS; expanded model of cultural intelligence; E-CQS; expatriation intention; NCA; necessary condition analysis; explanatory power; CQ sub-dimensions; cross-cultural competencies.

Reference to this paper should be made as follows: Richter, N.F., Schlaegel, C., van Bakel, M. and Engle, R.L. (2020) 'The expanded model of cultural intelligence and its explanatory power in the context of expatriation intention', European J. International Management, Vol. 14, No. 2, pp.381-419.

Biographical notes: Nicole Franziska Richter is an Associate Professor of International Business at SDU. Her research focuses on strategic and international management with a specific focus on cross-cultural aspects. She received her $\mathrm{PhD}$ from the University of Hamburg in 2009 and her habilitation (state doctorate) from TU Hamburg in 2015.

Christopher Schlaegel is an Assistant Professor of International Management at the University of Groningen, The Netherlands. His current research focuses on determinants and outcomes of intercultural competence (i.e. cultural intelligence) as well as substitutional and compensatory configurations with emotional intelligence. He received his $\mathrm{PhD}$ from Otto von Guericke University Magdeburg, Germany.

Marian van Bakel is an Associate Professor of International HRM at SDU. Her research focuses on international human resource management, particularly expatriate management and how expatriates connect with locals. She received her PhD from Radboud University Nijmegen, the Netherlands.

Robert L. Engle is Professor and Chair of International Business at Quinnipiac University. He teaches international management and cross-cultural research courses in both the graduate and undergraduate programs. He received his doctorate in management from Nova Southeastern University.

\section{Introduction}

The majority of researchers refer to cultural intelligence (CQ) as an individual's capability to adapt effectively to, and function effectively in, new cultural contexts (Earley and Ang, 2003; Andresen and Bergdolt, 2016). While various measures are available to assess CQ (e.g. Thomas et al., 2015; Alon et al., 2016), the CQ scale (Ang et al., 2007) is the measure that has been used most often in previous empirical studies (Matsumoto and Hwang, 2013). In this scale CQ is conceptualised as a multidimensional construct, consisting of four dimensions (i.e. cognitive CQ, metacognitive CQ, motivational CQ and behavioural CQ). Van Dyne et al. (2012) introduced a more finegrained measure of CQ, the 37-item expanded CQ scale (E-CQS), which decomposes the four primary $\mathrm{CQ}$ dimensions into 11 sub-dimensions, allowing for a more nuanced assessment of CQ. We argue that these more specific facets of CQ are better able to explain relevant outcomes and therewith show a higher potential for theory building in the field. To exemplify and evaluate the utility of the E-CQS for theorising in a crosscultural context, the present study examines the association between CQ and expatriation intention (EI). 
Expatriates, employees who are sent from their company to live and work in another country for a period of two or more years (Caligiuri, 2000), are seen as key in managing the international operations of firms (Collings et al., 2007), which is why understanding EI is so important (McEvoy and Buller, 2013). EI refers to an individual's anticipation and subjective probability to work as an expatriate in the future (e.g. Remhof et al., 2013; Engle et al., 2015). Authors have identified various determinants of EI. In addition to external factors related to the country and organisational environment of the EI endeavour (e.g. Bader et al., 2015; Pinto et al., 2017), studies have examined the association with personality, international experiences and CQ (e.g. Tharenou, 2003; Mol et al., 2009; Remhof et al., 2013). The collective results of prior studies indicate that individuals' CQ is positively associated with EI (Schlägel et al., 2017). Previous studies that used the CQ scale and the four CQ dimensions have shown that motivational CQ is most strongly associated with EI (Kim and Froese, 2012; Huff, 2013; Engle et al., 2015; Schlägel and Sarstedt, 2016). In contrast, there is less support for the association of other dimensions of CQ with EI, for instance metacognitive CQ.

Motivational CQ refers to individuals' capability to direct attention and energy towards learning about and functioning in situations that are characterised by cultural differences (Ang and Van Dyne, 2008). While there is a general consensus that motivation is a multidimensional construct that (at least) consists of intrinsic and extrinsic aspects (e.g. Cerasoli et al., 2014), in previous studies motivational CQ does not take into account this multidimensionality. From a theoretical perspective, this gap is important to fill because, in spite of the number of studies that have shown a clear positive association between motivational CQ and EI, we still lack a deeper understanding of the role that intrinsic and extrinsic motivations play in driving individuals' intention to work as an expatriate and how these might vary in different cultural contexts (see, for instance, the discussion in Haines et al., 2008). Likewise, metacognitive CQ comprises different mental processes, among them planning, awareness and checking which can be assumed to be of different relevance in the formation of intentions. While authors often argue that planning is of specific relevance in forming intentions, testing these arguments relied on testing the overall association between metacognitive CQ and EI, so far. Collapsing the multidimensional nature of these constructs into a unidimensional construct potentially may hinder a more finegrained exploration of associations and may therewith make theorising less accurate. Finally, it may lead practitioners in the wrong direction, as associations that are true for the overall construct might not necessarily be true for the sub-dimensions. Hence, our first and key contribution is that we advance the existing theoretical understanding of the formation of EI by emphasising the role of sub-dimensions of CQ. Doing so, we answer recent calls to deepen theory by more closely examining the specific components that constitute individuals' CQ (Gelfand et al., 2008).

Our second contribution is that we examine the predictive validity of the E-CQS and its sub-dimensions in predicting EI over and above well-established determinants of EI (i.e. personality traits, international experience and language ability), and compare the value of the E-CQS to the original CQS. We do this with reference to different countries or cultural contexts - which can be considered a third contribution. This is relevant for researchers aiming to use the E-CQS in their empirical models on EI and finally offers insights into the overall value of the scale in contrast to the original CQS and other predictors of EI. 
Based on samples of university business students from Germany, the USA and China, we assess the variance explained in EI by the E-CQS and the CQS and compare the structure and relevance of CQ (sub-)dimensions across the three countries. For this purpose, we make use of regression and necessary condition analyses to understand if the CQ (sub-)dimensions are relevant determinants of EI and if they might also be necessary conditions for EI to be present. We show that the E-CQS is of value when it comes to understanding EI as the sub-dimensions directly pick-up theoretical arguments implicitly outlined by researchers in previous studies and offer more specific and more actionable advice for managers. Our results demonstrate that the E-CQS has predictive validity above and beyond not only the CQS but above and beyond well-established determinants of EI. Finally, we show that the E-CQS can be used in different country settings and our results demonstrate the relevance to further explore country differences.

\section{CQ as a determinant of EI: literature review, theoretical background and hypotheses}

\subsection{A review of research on the expanded model of $C Q$}

According to Earley and Ang (2003), CQ comprises four key dimensions: cognitive CQ, metacognitive CQ, motivational $\mathrm{CQ}$ and behavioural CQ. Along with this conceptualisation, Ang and associates (Ang et al., 2006; Ang et al., 2007; Van Dyne et al., 2008) have developed a scale comprising a total of 20 items that represent these four dimensions, the CQ-scale (CQS) (see Table 1 for an overview of definitions and items). There are several other measurement instruments available to assess CQ (for an overview, see Matsumoto and Hwang, 2013). Most closely related to the CQS and extended CQS are the short form measure of CQ (SFCQ; Thomas et al., 2015; consisting of: cultural knowledge, cultural metacognition and cultural skills) and the business cultural intelligence (BCIQ; Alon et al., 2016; consisting of global knowledge, motivation, listening and communicative adaption, and cognitive preparation and learning behaviour). We decided to focus on the CQS and its extension as the scale combines all facets by which CQ is defined in the field (Earley and Ang, 2003; Andresen and Bergdolt, 2016) and the CQS is the scale used most often in existing studies (Matsumoto and Hwang, 2013).

Van Dyne and colleagues (Van Dyne et al., 2012) have recently expanded the scale and delineated 11 sub-dimensions (nested in the four primary dimensions) aiming to provide a deeper understanding of CQ and allowing for more refined theorising, and testing. For instance, they delineate two sub-dimensions of cognitive CQ: (1) culturegeneral knowledge of the universal elements that constitute formal and informal institutional environments (e.g. economics, politics and cultural values); (2) contextspecific knowledge about manifestations of cultural universals in a context and knowledge of how to be effective in this context (such as a country or subculture, e.g. the subculture of managers). In a similar fashion, they delineate sub-dimensions of the other primary dimensions of CQ: They specify three metacognitive mental processes, three sub-dimensions of motivational CQ and three sub-dimensions of behavioural CQ (Van Dyne et al., 2012). Table 1 provides the definitions and sample items for all subdimensions. 
Table 1 Expanded versus original CQ construct (and scale)

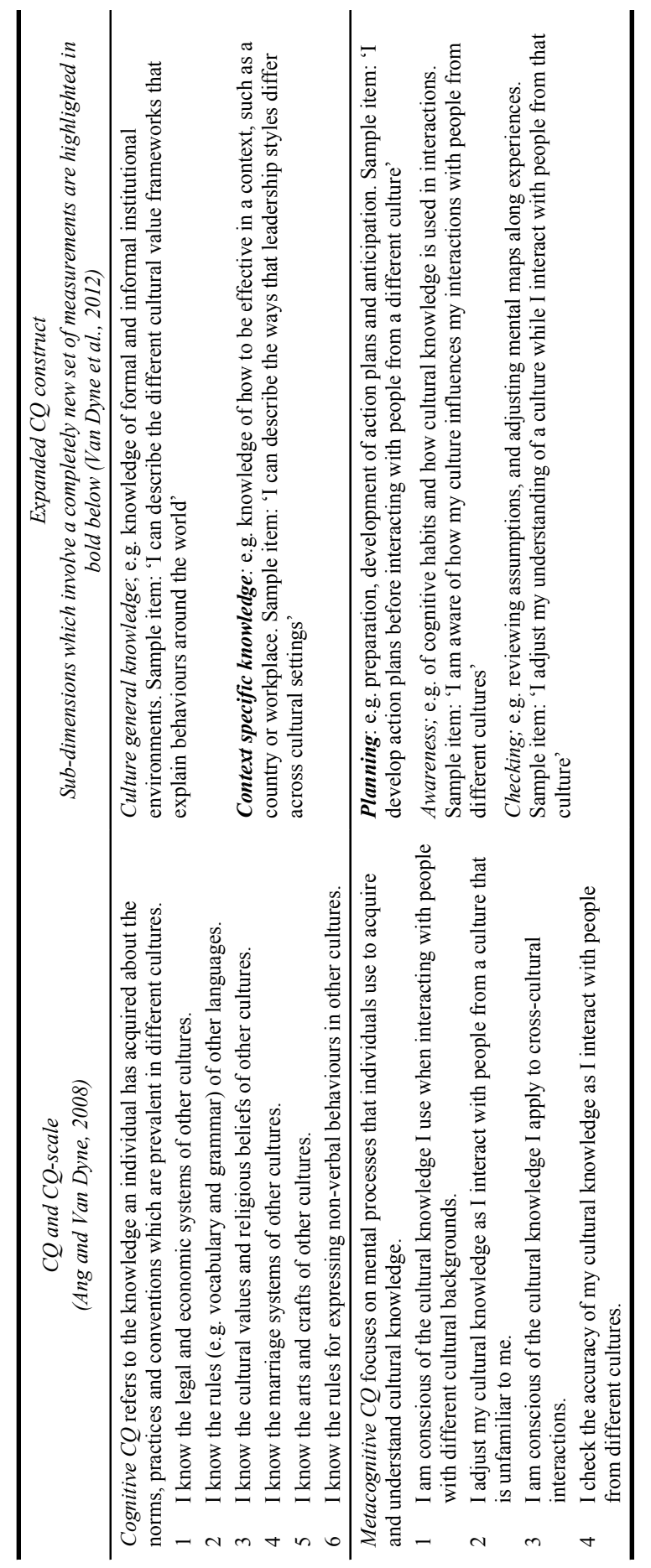


Table 1 Expanded versus original CQ construct (and scale) (continued)

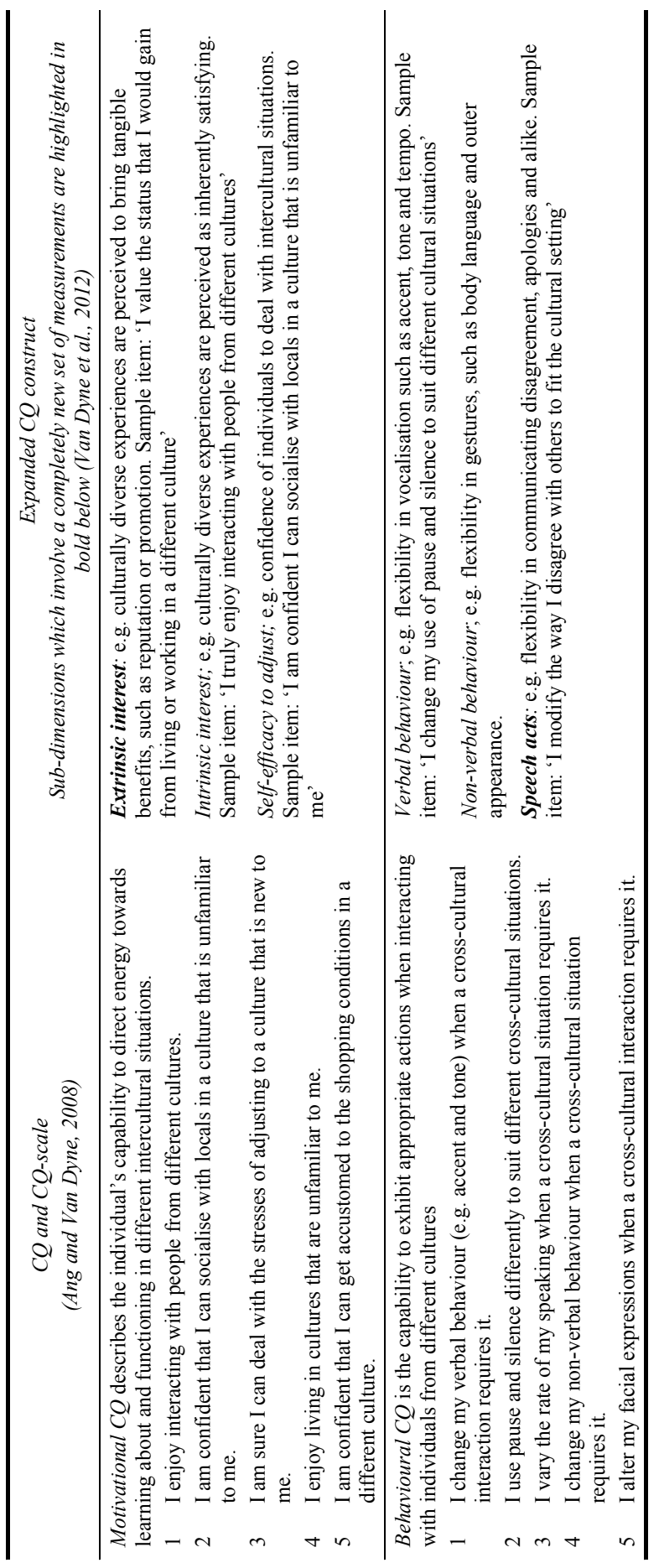


Along with this conceptualisation, Van Dyne et al. (2012) offer a set of items to operationalise the 11 sub-dimensions. Comparing the E-CQS with the 20-item CQS, we find that it supplements or slightly modifies items to measure seven of the subdimensions. However, it provides a completely new set of items for four sub-dimensions. This new set of items operationalises context-specific knowledge and relates to the management context. That is it refers to the knowledge of effective management practices across cultural contexts. Furthermore, the items operationalising planning (metacognitive CQ), extrinsic interest (motivational CQ) and speech acts (behavioural CQ) are new (see Table 1). Hence, the additional value of the instrument above and beyond the CQS in the context of EI can be assessed best by examining the four sub-dimensions which have not been included in the CQS.

A systematic review of previous research that cites Van Dyne et al. (2012), i.e. the article introducing the E-CQS, shows that studies that use the E-CQS for their empirical designs are still very scarce (see Table 2). In addition to the study by Van Dyne et al. (2012) who focused on testing the psychometric properties of the E-CQS, five other studies included (parts of) the E-CQS for testing its association with different crosscultural outcomes, such as leadership. None of the studies has used the E-CQS to test the relationship between CQ and EI. Four out of the five studies that make use of the E-CQS for their empirical models do not use the findings on the sub-dimensions of the scale, but generate interpretations on the level of the four primary dimensions or on the level of a specific primary dimension (Mor, 2013, Mor et al., 2013; Philippart, 2014; McComas, 2014; Rockstuhl et al., 2015,). The potential the scale might offer for a deeper understanding and more refined theorising is, thus, neglected.

Grubb (2015) who uses the E-CQS in researching the relationship between CQ and authentic leadership reports correlations for both the E-CQS and the CQS with leadership. He reports correlations between -0.07 and 0.18 for six sub-dimensions of the E-CQS with authentic leadership, which are lower than the correlations for some of the primary CQ dimensions with the leadership outcome. Still, with the design of the study, it is rather hard to understand the value that the E-CQS might provide to research and theorising. No study has systematically compared the predictive validity of the CQS versus the E-CQS. Given the increasing demands on the accuracy and parsimony of measures in international management research (e.g. Richter et al., 2016), it would be desirable to understand whether 17 additional items provides a deeper understanding of the CQ construct and its relationships with antecedents and outcomes. This also addresses questions on the applicability of the E-CQS in cross-cultural contexts, in terms of measurement invariance (to ensure that different country findings that might be generated are not a result of differences in measurement, Harzing et al., 2013), as well as evaluating if the instrument is able to explain phenomena in other cultural contexts (Tsui et al., 2007). 
Table 2 Overview of previous empirical studies that utilised the expanded CQ scale (E-CQS)

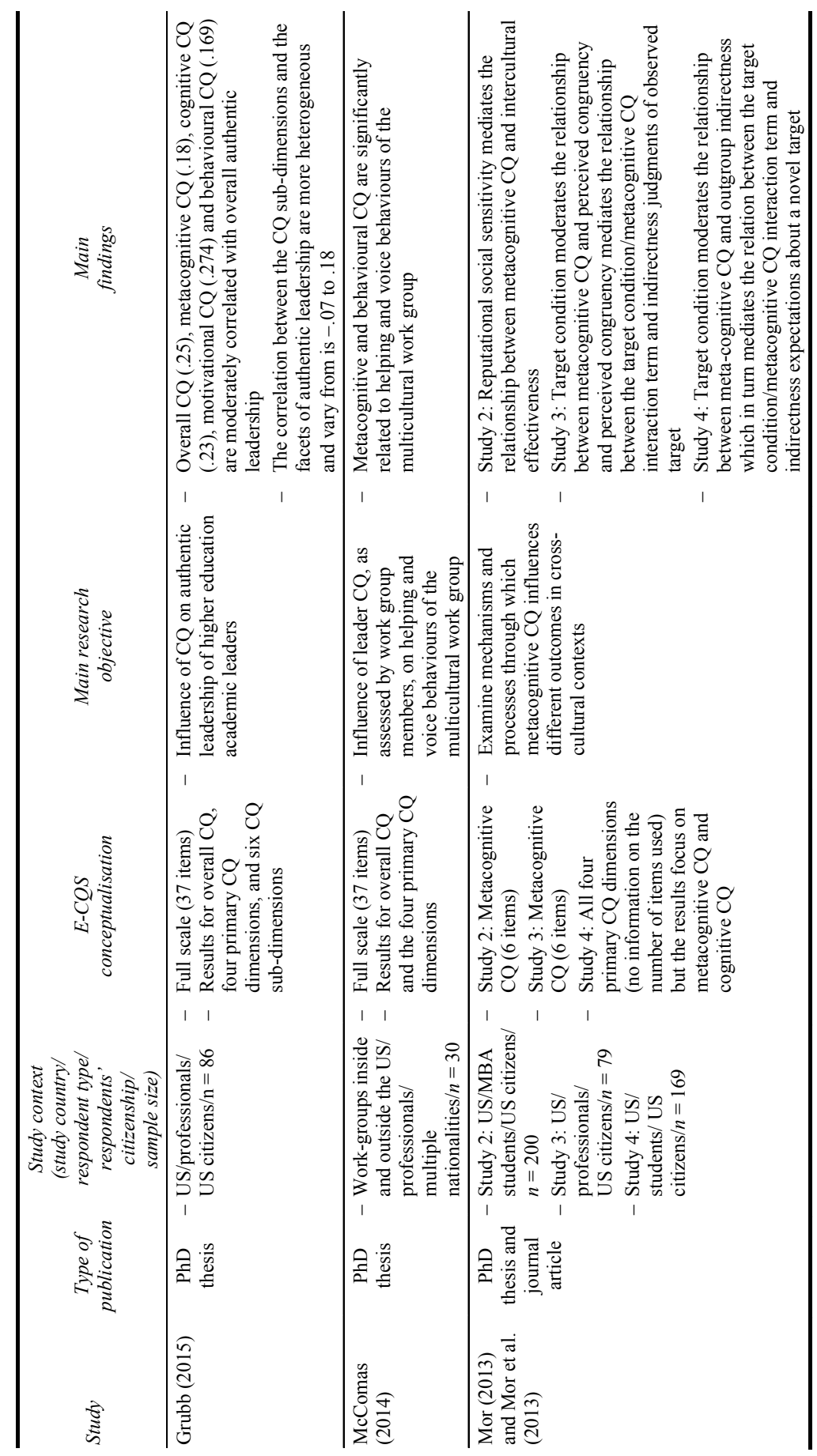


Table 2 Overview of previous empirical studies that utilised the expanded CQ scale (E-CQS) (continued)

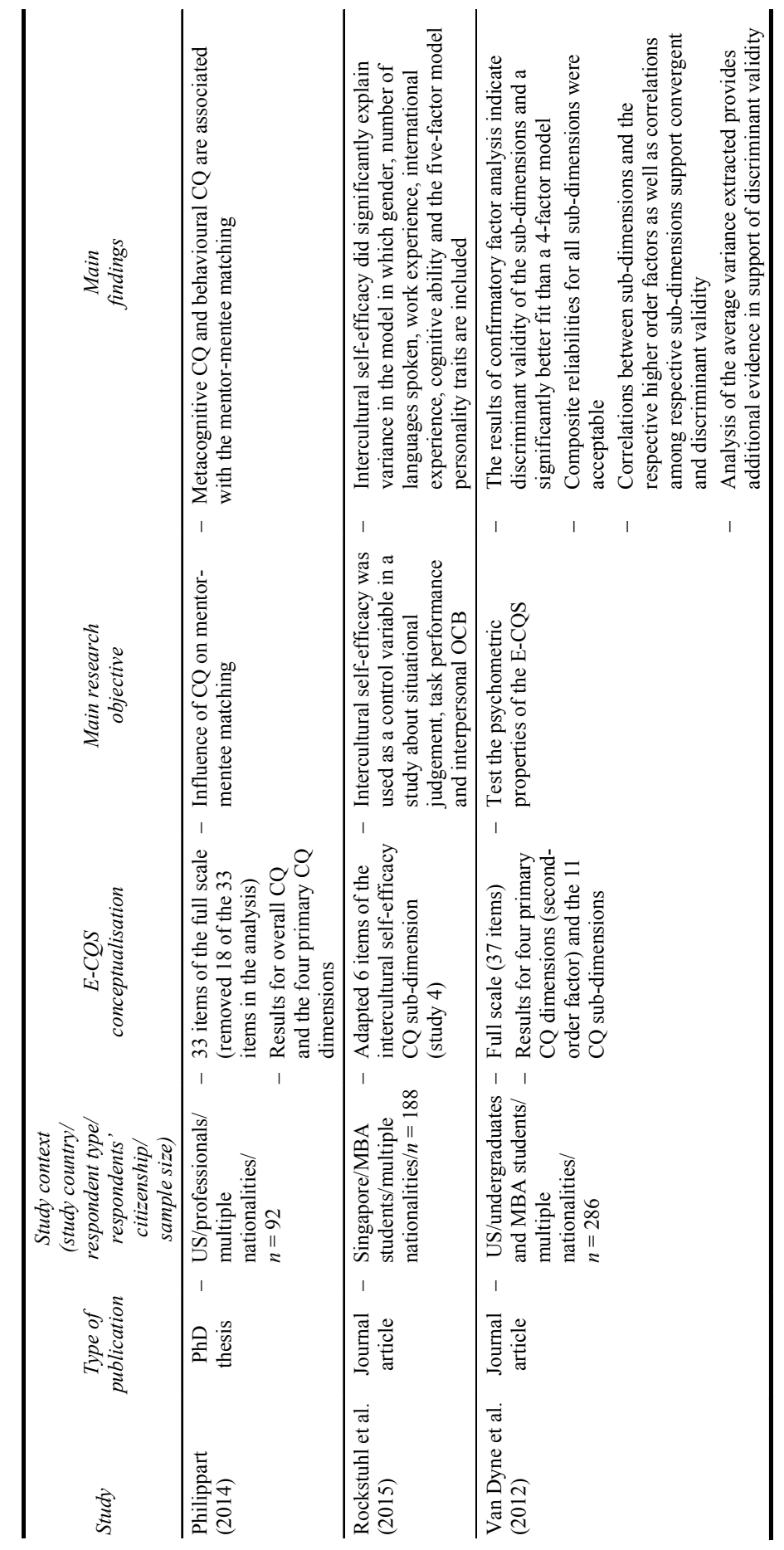


Table 3 Overview of quantitative empirical studies that examined the relationship between CQ and EI

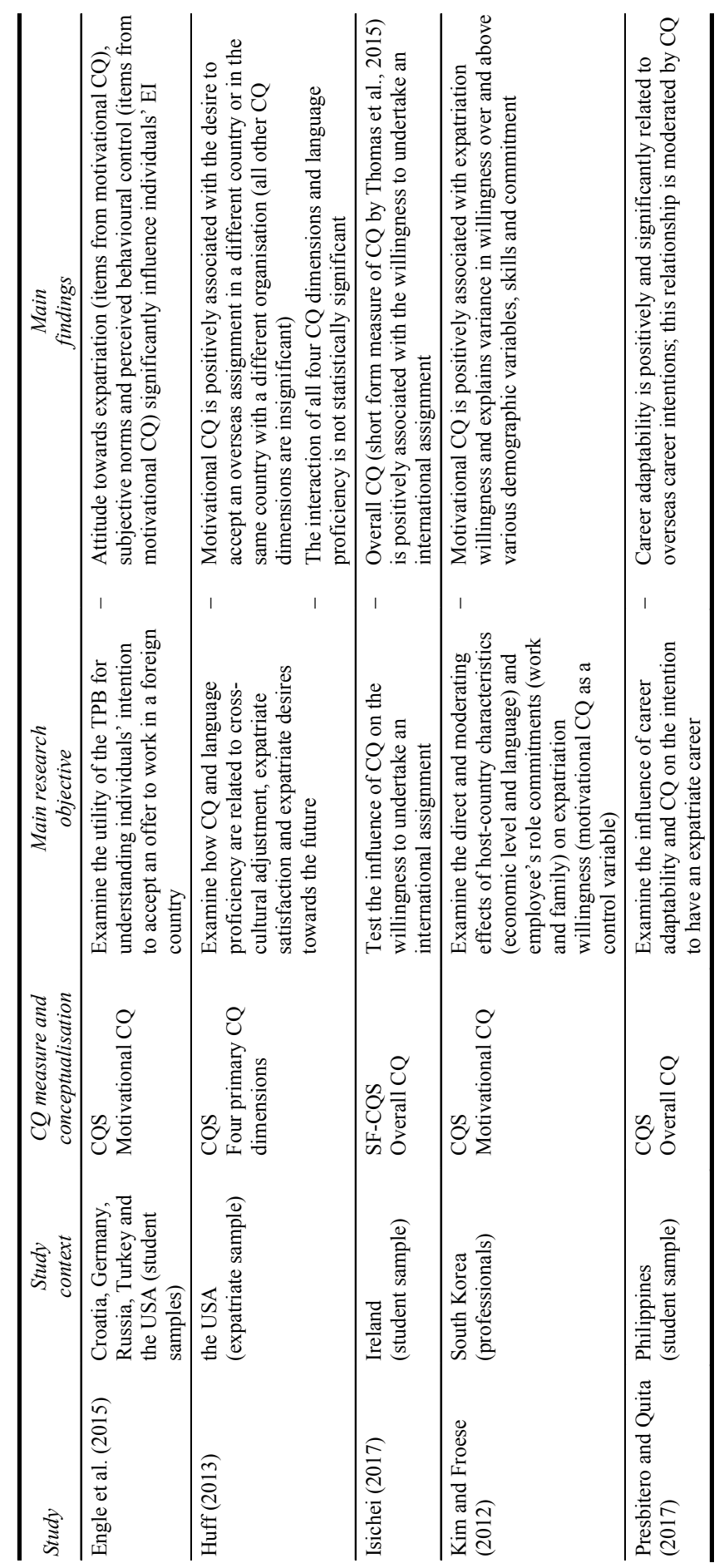


Table 3 Overview of quantitative empirical studies that examined the relationship between CQ and EI (continued)

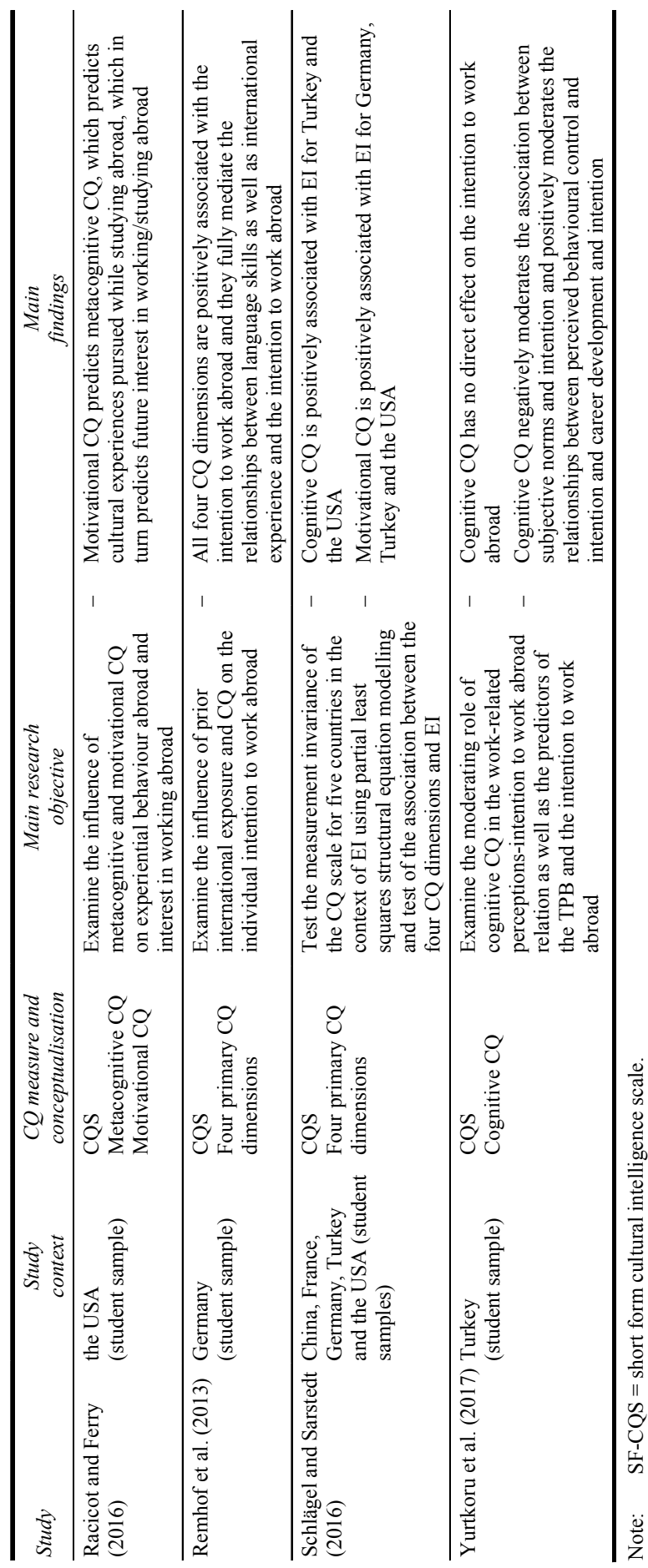




\subsection{A review of prior research on EI and the CQ-EI relationship}

Researchers for more than two decades have examined the determinants of EI (also referred to as expatriation willingness, and international relocation willingness). Previous studies in particular focused on the influence of personality traits (Mol et al., 2009), showing that openness to experience and extraversion are positively related to EI. Recently studies started to examine the influence of more narrow personality constructs, such as personal initiative (Baluku et al., 2018) and sensation seeking (Stoermer et al., 2017), on EI. Other studies (explicitly or by the inclusion as control variables) have examined the association between international experience and EI (Froese and Peltokorpi, 2013; Remhof et al., 2013) as well as between language proficiency (i.e. the number of languages an individual speaks) and EI (Froese and Peltokorpi, 2013; Huff, 2013; Engle et al., 2015), showing that both are positively associated with a higher EI. Finally, several studies explored the relationship between CQ and EI. In a systematic literature review of studies on the relationship between CQ and EI, we identified nine empirical studies on the CQ and EI relationship - none of these used the E-CQS. Table 3 provides a summary of these studies.

Two studies examined the relationship between overall CQ and EI basically providing support for a positive association of overall CQ and EI either directly or as a moderator (Isichei, 2017; Presbitero and Quita, 2017). The remaining seven studies refer to the primary CQ dimensions and either test the associations of all dimensions and EI (Huff, 2013; Remhof et al., 2013; Schlägel and Sarstedt, 2016) or only a specific dimension and EI (Kim and Froese, 2012; Engle et al., 2015; Racicot and Ferry, 2016; Yurtkoru et al., 2017). Results provide strong support for a positive association of motivational CQ with EI (six out of six studies that tested the association of motivational CQ with EI show a positive association). Likewise, the association of cognitive CQ with EI receives rather good support (two out of four studies show a positive association). The associations of metacognitive CQ and behavioural CQ with EI find less support (each with one out of three studies showing a positive association).

Seven of the articles reviewed are more comprehensively grounded in a theoretical framework. Two studies (Engle et al., 2015; Yurtkoru et al., 2017) used Ajzen's (1991) Theory of Planned Behaviour (TPB) that specifically aims at the explanation of individuals' intentions. Engle et al. (2015) applied specific items of the motivational CQ dimension to measure two of the three determinants of intention, namely attitude and perceived behavioural control. Yurtkoru et al. (2017) expanded the original TPB by adding cognitive CQ as a moderator of the antecedents of EI. Other authors (Savickas and Porfeli, 2012; Schlägel and Sarstedt, 2016; Presbitero and Quita, 2017) referred to the theory of successful intelligence (Sternberg, 1999) either individually or in combination with other theoretical frameworks, such as career construction theory. Finally, authors referred to theories of learning (Kolb, 1984; Bandura, 1986) in outlining arguments on the antecedents of EI (Remhof et al., 2013; Racicot and Ferry, 2016). Hence, only two studies used a theoretical framework that specifically aims at the explanation of individuals' intentions, the TPB.

While prior research has significantly contributed to a better understanding of the association between CQ and EI, the findings and theoretical mechanisms outlined are partly inconclusive and in an early stage of theorising. For instance, while we have learned that motivational CQ was positively related to EI, we still lack an in-depth understanding as to the extent intrinsic and extrinsic motivational factors are relevant in 
forming EI and how this understanding could best be used by managers (Ryan and Deci, 2000; Kanfer, 2012). Likewise, less support for the association between metacognitive CQ and EI does not necessarily mean that this translates into weak associations for all metacognitive sub-dimensions. On the contrary, there might be sub-dimensions of metacognitive CQ that can be hypothesised to be more strongly associated with EI. The E-CQS and the expanded conceptualisation of CQ allow for such detailed analyses, going beyond the four primary CQ dimensions (Van Dyne et al., 2012). In addition to analysing the predictive relevance of the E-CQS against the CQS on EI, we will also demonstrate whether the E-CQS shows predictive validity over and above established predictors of EI, such as personality traits, international experience and language ability.

\subsection{Research hypotheses on the relationship between $C Q$ and EI}

To derive hypotheses on the relationships between CQ (or the E-CQS) and EI, we make use of the TPB (Ajzen, 1991) and its three key determinants of intention. The first determinant of the TPB is an individual's attitude towards a behaviour and stems from an evaluation of positive and negative aspects related to the behaviour's expected value (Fishbein and Ajzen, 1975). Individuals will develop a higher intention to become an expatriate if they come to a positive evaluation of the potential benefits (e.g. recognition, further income and satisfaction) versus the potential costs (e.g. stress) related to the expatriation endeavour. The second determinant, the perceived behavioural control, is the perceived ease an individual attaches to the behaviour or the perceived control that a person feels to have over performing the behaviour (Ajzen, 1991). We posit that individuals who perceive that they have strong capabilities and skills to perform successfully as an expatriate in a different cultural context will have greater perceived behavioural control and a higher EI. Finally, subjective norm is referred to as the perceived (positive) opinions of significant others about the behaviour (Fishbein and Ajzen, 1975). Accordingly, we assume that if individuals believe significant others would value and encourage expatriation, and if they are motivated by these others, they will show a higher EI.

Using the TPB model, we assume that cognitive CQ, metacognitive and behavioural CQ will be positively associated with EI as they imply a higher perceived behavioural control of individuals. Moreover, we assume that motivational CQ will be positively associated with EI, as it implies a more positive attitude towards the behaviour, a higher perceived behavioural control and subjective norm. We furthermore assume that as the (sub-)dimensions of CQ have different relevance before, during and after intercultural interactions take place, the strengths of their associations with EI might differ accordingly. Cognitive CQ is of specific relevance before and during the intercultural interaction, metacognitive CQ in all three phases (planning is of specific relevance before, awareness during and checking after the intercultural interaction). Motivational $\mathrm{CQ}$ is of specific relevance before and during the intercultural interaction. Behavioural $\mathrm{CQ}$ has specific relevance during the intercultural interaction. Following this classification, we assume that the sub-dimensions that are of specific relevance before the intercultural interaction takes place, i.e. during the phase of forming intentions, have most relevance in increasing EI.

We assume that a high cognitive CQ helps individuals to properly map new situations in culturally different countries and will, therefore, reduce their perceived uncertainty about a cross-cultural endeavour; individuals might perceive the cultural knowledge as a 
form of behavioural control. We argue that this is true for culture-general knowledge regarding the political and economic systems in other cultures, their languages, as well as their norms and values (see also Schlägel and Sarstedt, 2016). We, moreover, argue that the knowledge of how to be effective in a managerial context is relevant to increasing EI as it implies greater perceived behavioural control. Therewith, we hypothesise that the expanded CQ model comprising both forms of knowledge will be better able to explain $\mathrm{EI}$ as compared to the original CQS.

The literature suggests that a high metacognitive ability can result in the ability to learn new behaviours and adapt to change, while also specifically suggesting that expatriates' metacognitive skills contribute not only to the ability to cope with demands of their jobs but also contribute to higher EI (Schlägel and Sarstedt, 2016). Some authors more specifically focus on the aspect of planning, arguing that understanding the importance of preparation and planning, which is part of the primary metacognitive CQ dimensions, may advance the intention to work abroad (Remhof et al., 2013). In accordance with this thinking, we assume that metacognitive CQ will be positively associated with the intention to become an expatriate as it implies an increased perceived behavioural control. We, moreover, hypothesise that the expanded CQ model will be a better predictor of EI as compared to the original scale, as it more specifically relates to the process of planning. Planning skills may be engaged prior to intercultural interactions taking place, i.e. during the phase of forming intentions (Van Dyne et al., 2012). We assume that this mental process of planning is most strongly related to increasing the perceived behavioural control of individuals and therewith to increasing EI.

Motivational CQ is hypothesised to be the most important predictor of EI (see also Remhof et al., 2013). Authors argue that individuals with a high motivational CQ are intrinsically motivated to explore diverse cultural situations and enjoy new experiences (Remhof et al., 2013). We follow this argument and assume that individuals who enjoy living in other cultures and interaction with others will have a greater need and drive to do so, deriving an intrinsic benefit from these cultural experiences resulting in less stress (for the argument on stress, see also Templer et al., 2006; Chen et al., 2014; Crowne and Engle, 2016). Therewith, individuals with a high intrinsic motivational CQ will come to a more positive attitude towards the endeavour and to a higher EI. Engle et al. (2015) formalises this association, by using items related to intrinsic motivational CQ from the CQS to measure the attitude towards the behaviour in their empirical model. Research on work motivation also supports the idea of a strong relation between intrinsic interest (in general) and EI (Haines et al., 2008). Moreover, we assume that individuals who are more confident in their ability to interact with culturally different others, i.e. with a higher self-efficacy, will have a higher EI. Self-efficacy to adjust is a concept strongly related to the perceived behavioural control and is assumed to be a strong determinant of EI (see also Engle et al., 2015 who again used items from the motivational dimension of the CQS to operationalise perceived behavioural control).

Research shows that also extrinsic rewards are of relevance in the formation of EI (e.g. Haines et al., 2008) and demonstrates that the importance of extrinsic as compared to intrinsic motivators differs considerably in different cultures for diverse work-related outcomes (e.g. Hauff et al., 2015). We assume that a high extrinsic motivational CQ is associated with a more positive evaluation of the benefits of the endeavour, for instance, in the form of higher income and career opportunities. Hence, we assume that it increases EI via a more positive attitude towards the behaviour and that it is associated with gaining a reputation benefit among relevant others and therewith is associated with a 
higher EI through a more positive subjective norm. Hence, we suggest that motivational CQ is positively associated with EI. Moreover, we posit that the expanded CQ concept, comprising extrinsic interest as a new and additional facet, will be better able to explain EI as compared to the original scale.

Finally, behavioural $\mathrm{CQ}$ is hypothesised to be positively associated with EI. Behavioural CQ becomes most relevant in the actual intercultural interaction and the performance of an individual, hence, we assume that it might show less relevance before engaging in intercultural interactions, i.e. during the phase of developing intentions. Still, we assume that it contributes to the perceived behavioural control of an individual and might therefore contribute to a higher intention to work as an expatriate. While the expanded scale comprises another facet of behavioural control, namely speech acts, we assume that this might contribute to a more fine-grained interpretation, yet we do not expect it to be a major factor in increasing explanatory power in the context of EI.

From the above arguments, we can only tentatively assume which of the introduced sub-dimensions might have the strongest association with EI as we borrow arguments from related fields. Therefore, we will refrain from outlining specific hypotheses on the effect of the individual sub-dimensions, but comment on their individual contribution in the discussion section to advance theorising. Hence, we propose the following two research hypotheses:

Hypothesis 1: The four primary $C Q$ dimensions (cognitive $C Q$, metacognitive $C Q$, motivational $C Q$ and behavioural $C Q$ ) are positively associated with EI.

Hypothesis 2: The expanded model of $C Q$ is better able to explain EI than the original model of $C Q$.

\section{Methodology}

\subsection{Sample and data collection}

We tested our hypotheses using data collected among management and economics students at the bachelor and master level at universities in Germany, the USA and China by means of a paper and pencil survey. We selected these countries as they represent different informal (i.e. cultural values) and formal (i.e. laws and regulations) institutional environments, which influence individuals' intentions and behaviour. For example, the countries represent three different cultural clusters (Ronen and Shenkar, 2013), i.e. Confucian Asia (China), Germanic (Germany) and Anglo (the USA), with different cultural values that, for instance, may influence whether an individual is willing to leave parents, family and friends behind to accept an expatriate position. Student samples are controversially discussed in the literature (Peterson and Merunka, 2014; Ford, 2016). However, in the context of the present study they can be considered appropriate as they help to isolate the potential influence of the national context and they allow to examine the phenomenon under study at the time the respondents actually build these specific intentions, i.e. students facing important career decisions (Bello et al., 2009).

In all countries, surveys were distributed within university lectures and completed in class, ensuring consistency across samples in terms of survey formats and data collection procedure (Leung, 2008). Participation was voluntary and answers were treated anonymously. The data collection resulted in 850 useable responses (Germany $N=360$, 
the USA $N=230$ and China $N=260$ ) and Tables A1-A3 provide descriptive statistics on the country samples. The average age of respondents for the German sample was 22 years $(57 \%$ were female), 21 years $(66 \%$ were female) for the US sample and 25 years ( $73 \%$ were females) for the Chinese sample.

\subsection{Measures}

The measures used in our survey were taken from the literature and the survey was conducted in English in the USA, in Chinese in China and in German among the German respondents. We followed Brislin (1980) and used a translation-back-translation procedure to translate the English items from the literature into the native languages. Table 4 provides loadings, average variances extracted, Cronbach's alpha and the composite reliabilities, which are satisfactory for all constructs.

- Expatriation intention: We used three items taken from previous research (Engle et al., 2015) to measure EI. An example item is: 'To what extent have you considered working in a foreign country?' $(1=$ very little, $6=$ very great $)$.

- Cultural intelligence: We utilised the E-CQS involving 37 items all measured on a seven-point likert scale from $1=$ strongly disagree to $7=$ strongly agree. These 37 items operationalise the 11 sub-dimensions of CQ introduced by Van Dyne et al. (2012). There are 15 items which show an overlap with items from the original CQ scale by Ang and Van Dyne (2008); we provide examples of items in Table 1 (the full scale is protected by copyright, but can be requested at cquery@culturalq.com).

o Cognitive CQ: Culture-general knowledge is measured by five items. Contextspecific knowledge is measured using five items. The five items measuring culture-general knowledge show a strong overlap with the measures operationalising cognitive CQ in the original scale. There are, however, no items involved in the original CQS that refer to context-specific knowledge.

o Metacognitive CQ: Answers on three items are collected to measure the (newly operationalised) planning sub-dimension. Awareness is measured with three items. Finally, checking is measured by three items, two of which with strong overlap to the items in the original CQS.

o Motivational CQ: Three items pertained to intrinsic interest and in parts very high overlap to the CQS. The survey contained three items for extrinsic interest, which was not operationalised in the original CQS. Self-efficacy to adjust also contained three items and has items that are almost identical to the ones in the CQS.

o Behavioural CQ: The survey measured verbal behaviour with three items. Three items formed the highly reliable sub-dimension of non-verbal behaviour with one item in the original CQS which closely corresponds to an item of the expanded scale. The final sub-dimension was speech acts. Three items measured this sub-dimension. We did not identify any items in the original version of the CQS that are comparable to those that assess speech acts. 
Table 4 Scales and measurements

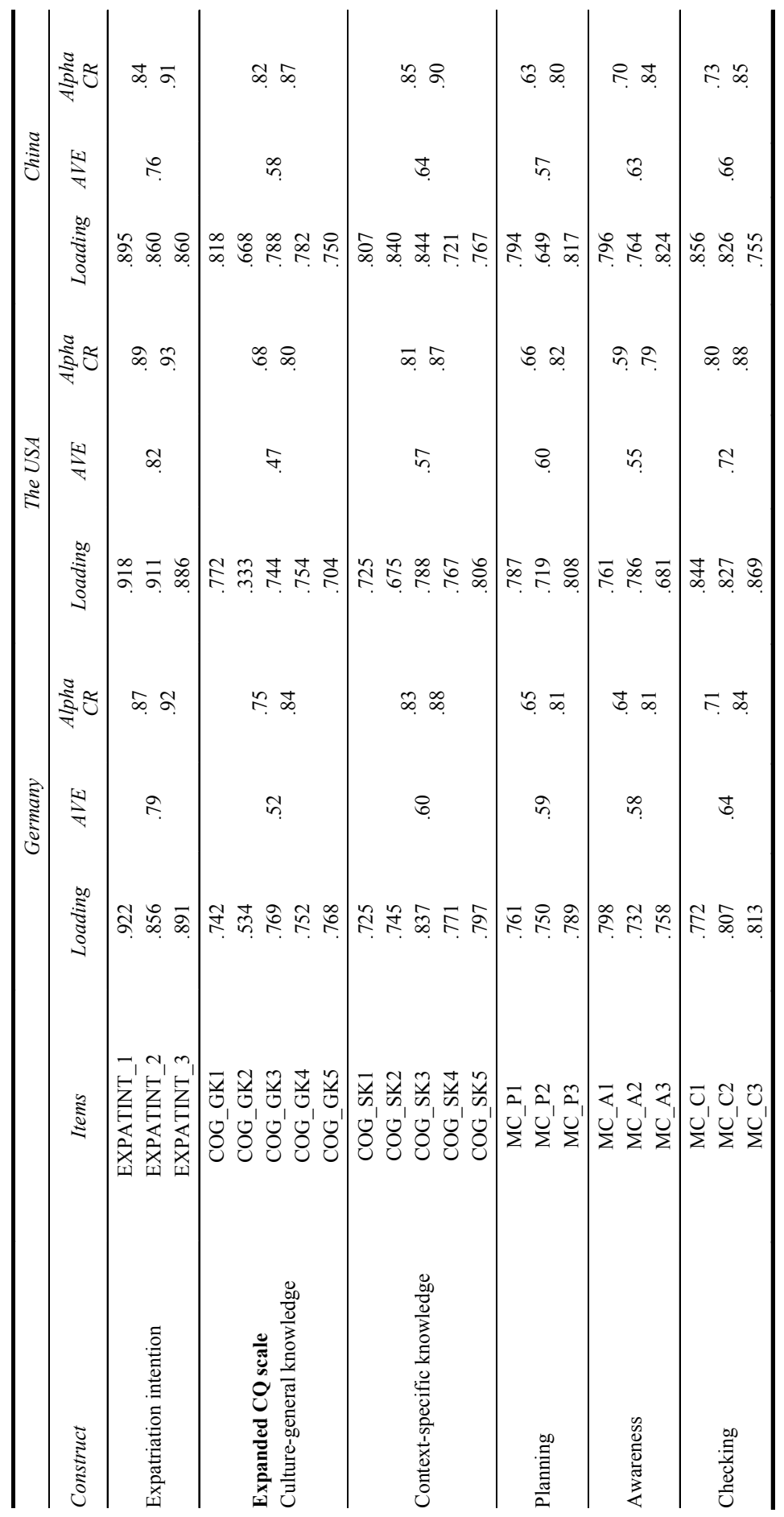


Table 4 Scales and measurements (continued)

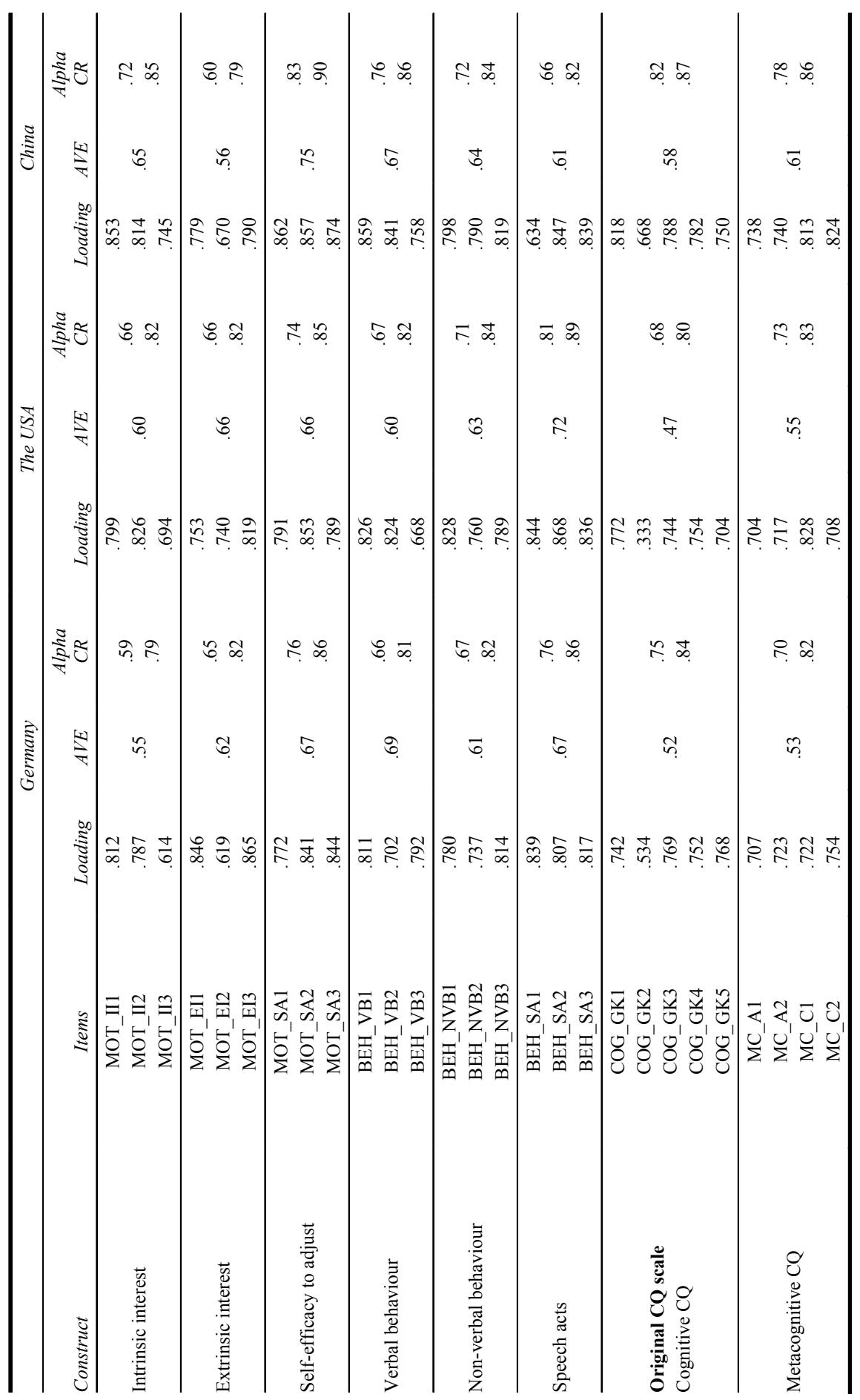


Table 4 Scales and measurements (continued)

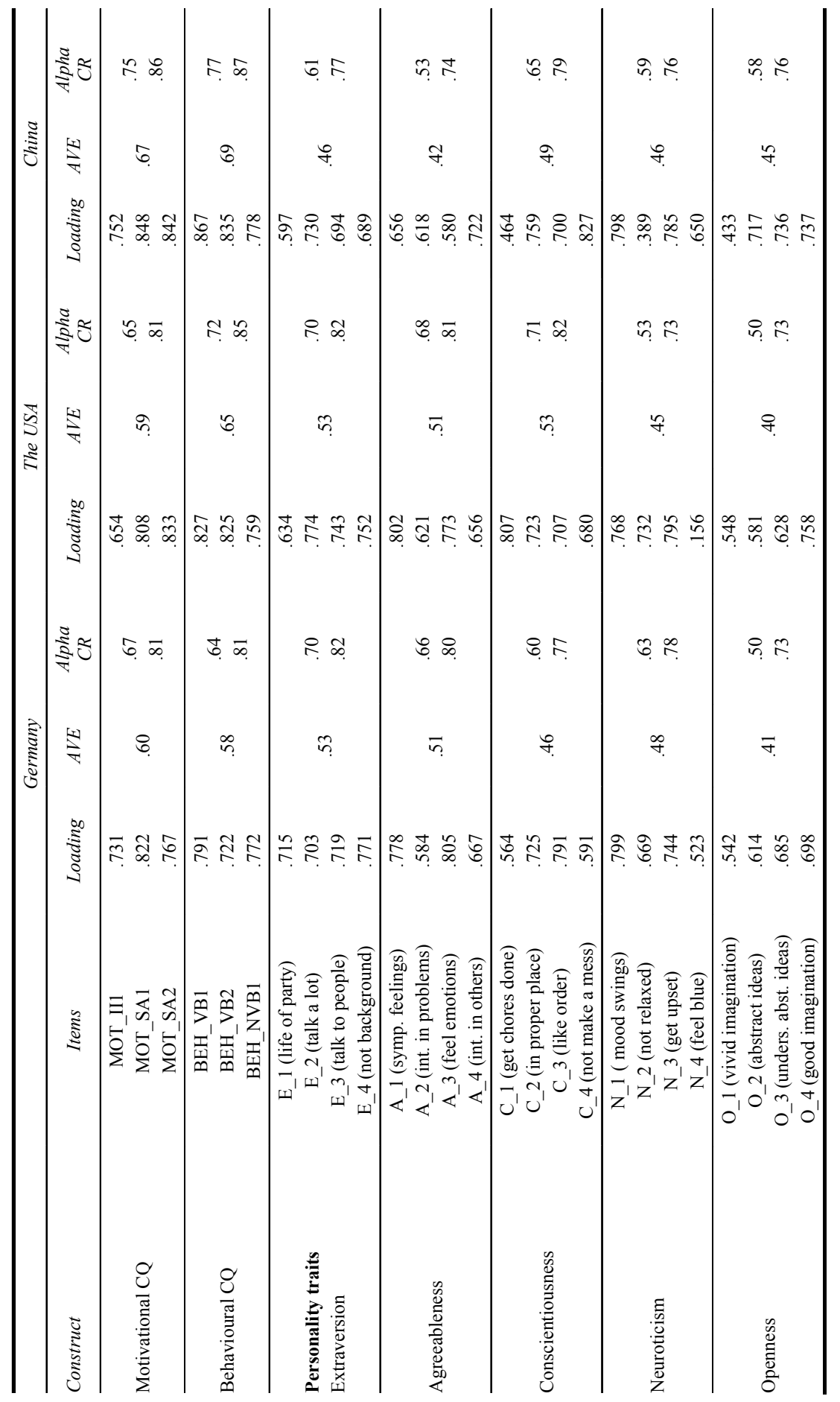


In addition to the above evaluations, we have tested the discriminant validity of the subdimensions using the heterotrait-monotrait ratio (HTMT). Along this criterion, constructs show issues of discriminant validity, if their HTMT is above .90 and if the $95 \%$ bootstrap confidence intervals of HTMT values do contain the value 1 (Hair et al., 2017). In all three countries, the sub-dimensions of behavioural CQ show issues of discriminant validity. In Germany, it is the new sub-dimension speech acts that does not show discriminant validity from non-verbal behavioural CQ. In the USA and likewise in China, the HTMT criterion is not met for speech acts and non-verbal, and also not for verbal and non-verbal behavioural CQ. Furthermore, the behavioural sub-dimensions of CQ also do not show discriminant validity against some of the metacognitive CQ subdimensions (for non-verbal behaviour and speech acts, the HTMT criterion is not met for awareness and checking in China; in the USA, planning and verbal behavioural CQ do not show enough discriminant validity).

We measured the four primary dimensions of $C Q$, namely cognitive $C Q$, metacognitive $\mathrm{CQ}$, motivational $\mathrm{CQ}$ and behavioural $\mathrm{CQ}$, using a selection of items from the E-CQS that correspond to the original CQS items. Thus, we do not use the original 20 item CQ scale in the comparison, which represents a limitation of the present study that we further elaborate on in the limitations section. An evaluation of the composite reliabilities of these measurements shows that all dimensions show satisfactory reliability for the three countries (see Table 4) and all four primary dimensions show discriminant validity along the HTMT criterion.

- Control variables: We included several control variables to ensure that any relationships found between CQ dimensions and EI are not confounded by differences in individual characteristics and to test the incremental predictive validity of the CQ dimensions over and above more established predictors of EI. We controlled for age (measured in years) as some studies found a negative relation between age and EI (e.g. Kim and Froese, 2012). We also controlled for gender (dummy coded female $=1$ and male $=0$ ) as previous studies found women less often intended to work as an expatriate compared to men (Engle et al., 2015). We also controlled for education (the number of semesters) as prior studies found a negative relation between educational level and EI (Engle et al., 2015). Several studies found a positive association between language ability (number of languages spoken except for native language) as well as international experiences (number of trips to foreign countries and number of weeks spent in foreign countries) and EI (Huff, 2013; Remhof et al., 2013). Therefore, we included both as control variables. Finally, we included the five-factor model of personality traits using the scale proposed by Donnellan et al. (2006), which shows satisfactory reliability for all items in all countries (see Table 4). Several studies found relations between personality traits and EI (Mol et al., 2009). Tables A1, A2 and A3 in the appendix provide an overview of mean values, standard deviations and correlations among all variables used in the three countries. 


\subsection{Analyses}

The following steps were undertaken to account for potential common method bias. First, survey items related to the dependent and the independent variables were separated within the survey and randomised within blocks to reduce a potential bias from their sequencing. Second, we assessed the potential influence of common method bias posthoc by using Harman's single factor test (Podsakoff and Organ, 1986) suggesting that there is no 'general factor' in the data. Hence, we are confident that common method bias is not a serious problem in our study.

We tested for measurement invariance between the country samples using the measurement invariance of composite models approach (see Henseler et al., 2016; Schlägel and Sarstedt, 2016). For this purpose, we made use of a partial least squares structural equation model and SmartPLS 3.0 (Ringle et al., 2015). The analysis showed that while we have established configural invariance, we do not have established compositional invariance. Hence, we do not have partial (and accordingly not full) measurement invariance (Hair et al., 2017). Therewith, we will refrain from comparing standardised coefficients across the country samples but interpret the results for the countries individually.

To test our hypotheses, we make use of two procedures. First, we make use of ordinary OLS regression analysis using IBM SPSS to identify the influence that our control and research variables assert on EI. These regression analyses involve three steps. In the first step, we evaluate the control model looking at the association of our control variables and EI. In the second step, we assess the (additional) associations of the $11 \mathrm{CQ}$ sub-dimensions and EI using regression analyses. In third step, we assess the association between the four primary CQ dimensions and EI (i.e. the explained variance above the control model) (see Figure 1). We test whether the E-CQS model has a significantly higher explanatory power as compared to the CQS model utilising Steiger's $Z$ (Steiger, 1980; Hoerger, 2013). ${ }^{1}$ For all models, we tested for multicollinearity referring to the variance inflation factors (VIF), especially in light of sub-dimensions of the E-CQS not meeting discriminant validity criteria. None of the VIF indicated problems of multicollinearity (highest VIF for Germany: 2.96 for speech acts; the USA: 3.36 for speech acts; China: 3.82 for non-verbal behaviour); we therefore refrain from eliminating constructs, yet also do not emphasise interpretation of findings for the sub-dimensions of behavioural CQ.

Second, we perform necessary condition analyses (NCA) on all determinants of EI using $R$ and the package NCA (Dul, 2016b; Dul, 2018) to complement the traditional regression approach. While our regression analysis provides information on the strength and direction of the relationship between different CQ dimensions and EI, the NCA tests whether specific CQ dimensions are necessary determinants of EI. Without the necessary determinant (e.g. CQ), the outcome (e.g. EI) will not exist (Dul, 2016a). As Dul (2016a) formulates it: '.. a necessary cause is a constraint... that must be managed to allow a desired outcome to exist'. The absence of a necessary condition cannot be compensated by other determinants, hence in its absence there is no EI. If we, for instance, find that extrinsic interest is a necessary condition for EI, EI will not exist without extrinsic interest being present. Hence, the NCA provides a valuable further understanding of the relevance of CQ sub-dimensions and answers recent calls to assess necessary conditions in the field (Rockstuhl and Van Dyne, 2018). 
Figure 1 Conceptual models and analysis approach

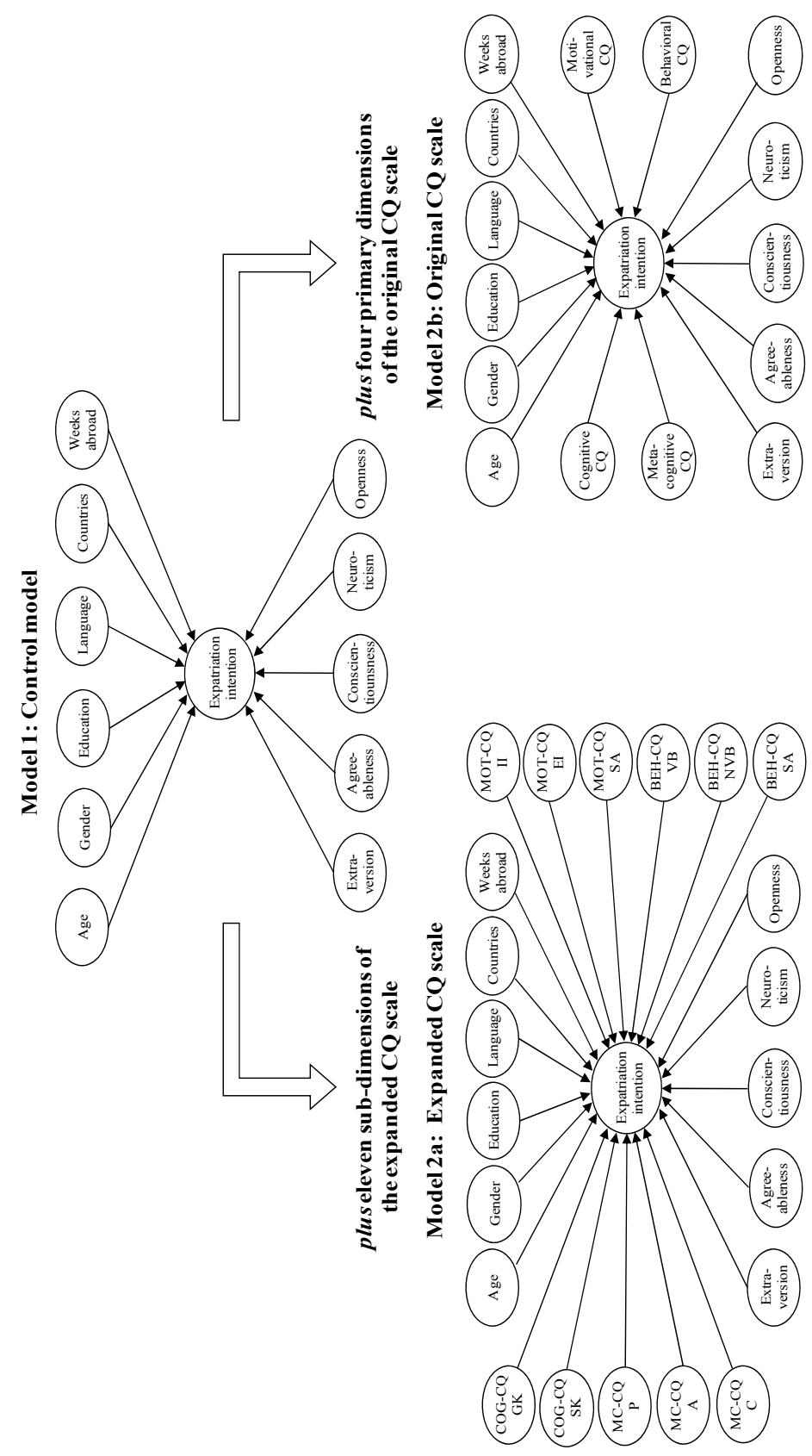


All analyses make use of the single item measures and factor scores derived by means of factor analyses. The above analyses enable us to gain an understanding of (a) the value that the sub-dimensions have for influencing EI. For this purpose, we will look at the regression coefficients and their statistical significance to assess the strengths of the association between each sub-dimension and EI. Furthermore, we will assess whether a sub-dimension is a necessary determinant of EI looking at the effect sizes obtained in the NCA and their statistical significance (Dul et al., 2018). Moreover, the above analyses enable us to gain an understanding of (b) the value that the expanded scale has above the original CQ scale for explaining variance in EI. For this purpose, we will compare the explanatory power of the model involving the E-CQS versus the model involving the CQS and evaluate whether it has significantly higher explanatory power (using Steiger's Z).

\section{Results}

\subsection{Results for the German sample}

First, we provide the results for the German sample. Table 5 provides an overview of the regression results for the control model (Model 1), the expanded CQS model (Model 2a) and the model for the four primary CQ dimensions (Model 2b). Furthermore, it presents the results of the NCA performed for all determinants of EI, more specifically it presents the effect sizes gained. Dul (2016b, p.30) offers the following benchmarks to interpret these effect sizes: $0<d<0.1$ represents a small effect, $0.1 \leq d<0.3$ a medium effect, $0.3 \leq d<0.5$ a large effect and $d \geq 0.5$ a very large effect.

The control model has a moderate explanatory power $\left(R^{2}=.17 ; R_{\text {adjusted }}^{2} .15\right)$. In Model $2 \mathrm{a}$, the explanatory power is significantly increased as compared to the control model $\left(R^{2}=.38 ; R_{\text {adjusted }}^{2}=.33\right)$. The sub-dimensions of CQ which significantly determine EI all belong to motivational CQ: intrinsic interest $(\beta=.20 ; p=.001)$, extrinsic interest $(\beta=.26 ; p=.000)$ and self-efficacy to adjust $(\beta=.16 ; p=.006)$ are significant positive drivers of EI. In Model $2 b$, we add the four primary CQ dimensions as defined in the original CQ scale to the control model. This increases the amount of explained variance as compared to the control model significantly $\left(R^{2}=.30 ; R_{\text {adjusted }}^{2}=\right.$ .27), however, also shows less explanatory power against the expanded CQ model. A Steiger's $Z$ test shows that the $R^{2}$-values between Models $2 \mathrm{a}$ and $2 \mathrm{~b}$ differ significantly, i.e. the expanded CQ model explains EI significantly better than Model $2 \mathrm{~b}$ only involving the four primary CQ dimensions $(Z=3.02 ; p=.002)$. Among the four dimensions of $C Q$, two are positive significant determinants of EI: metacognitive CQ $(\beta=.16 ; p=.009)$ and motivational CQ $(\beta=.31 ; p=.000)$.

From the NCA we find that some of the CQ dimensions and sub-dimensions are necessary conditions for EI, and some are not. For instance, intrinsic interest qualifies as a necessary condition, whereas extrinsic interest does not, although the latter is a relevant determinant for EI as found in the regression analyses. Hence, in the absence of intrinsic interest, there will be no EI. Only, if an individual has intrinsic interest, extrinsic interest can further increase the EI. Among the 11 sub-dimensions of CQ, the following qualify as medium high and significant necessary conditions to EI: checking as part of metacognitive CQ $(d=.14 ; p=.000)$, intrinsic interest $(d=.16 ; p=.000)$ and selfefficacy to adjust $(d=.15 ; p=.015)$. Finally, among the four original dimensions of the CQ model, two show a medium high and significant necessary condition effect size: metacognitive CQ $(d=.14 ; p=.000)$ and motivational CQ $(d=.14 ; p=.014)$. 
Table 5 Results of regression analysis and necessary condition analysis for EI

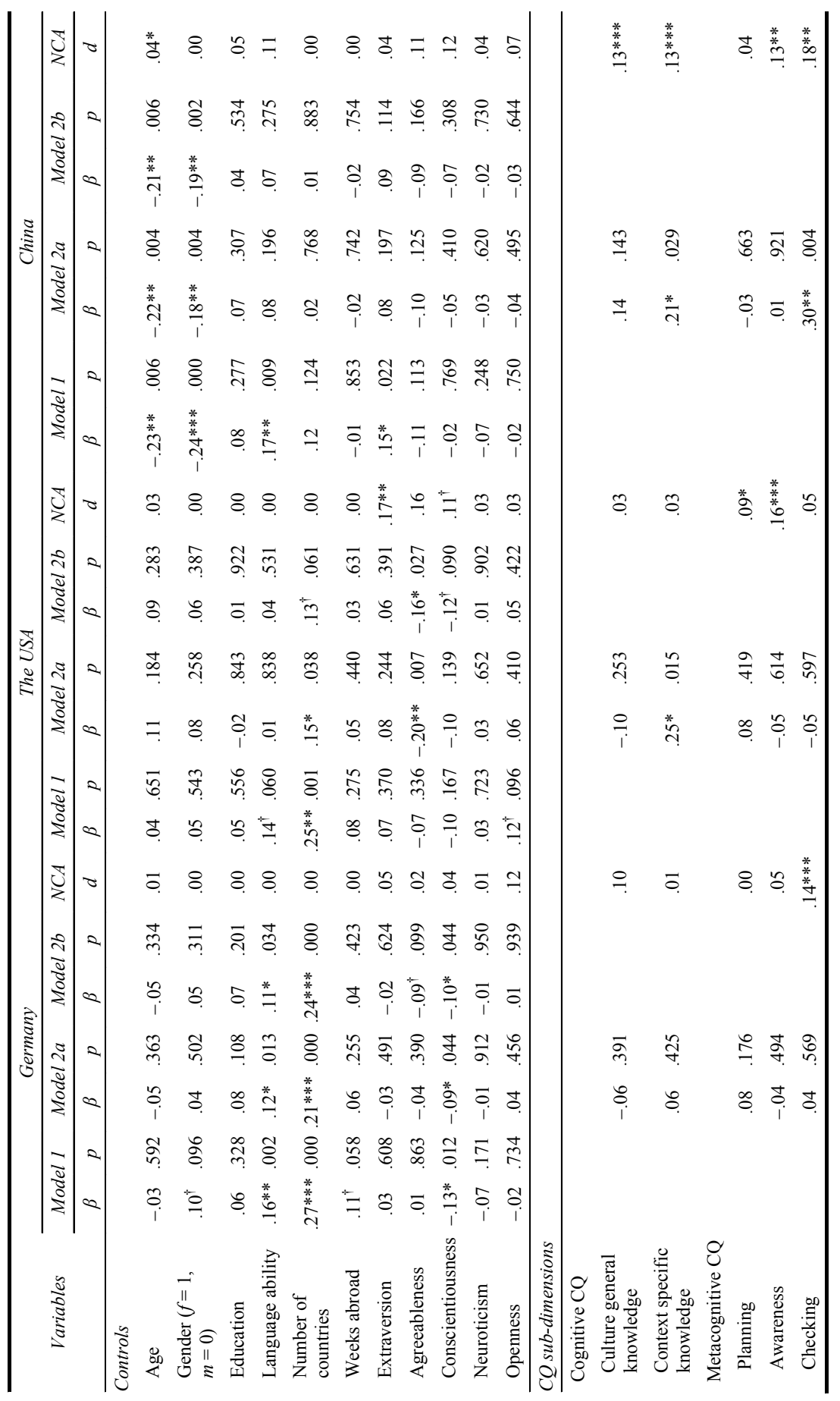


Table 5 Results of regression analysis and necessary condition analysis for EI (continued)

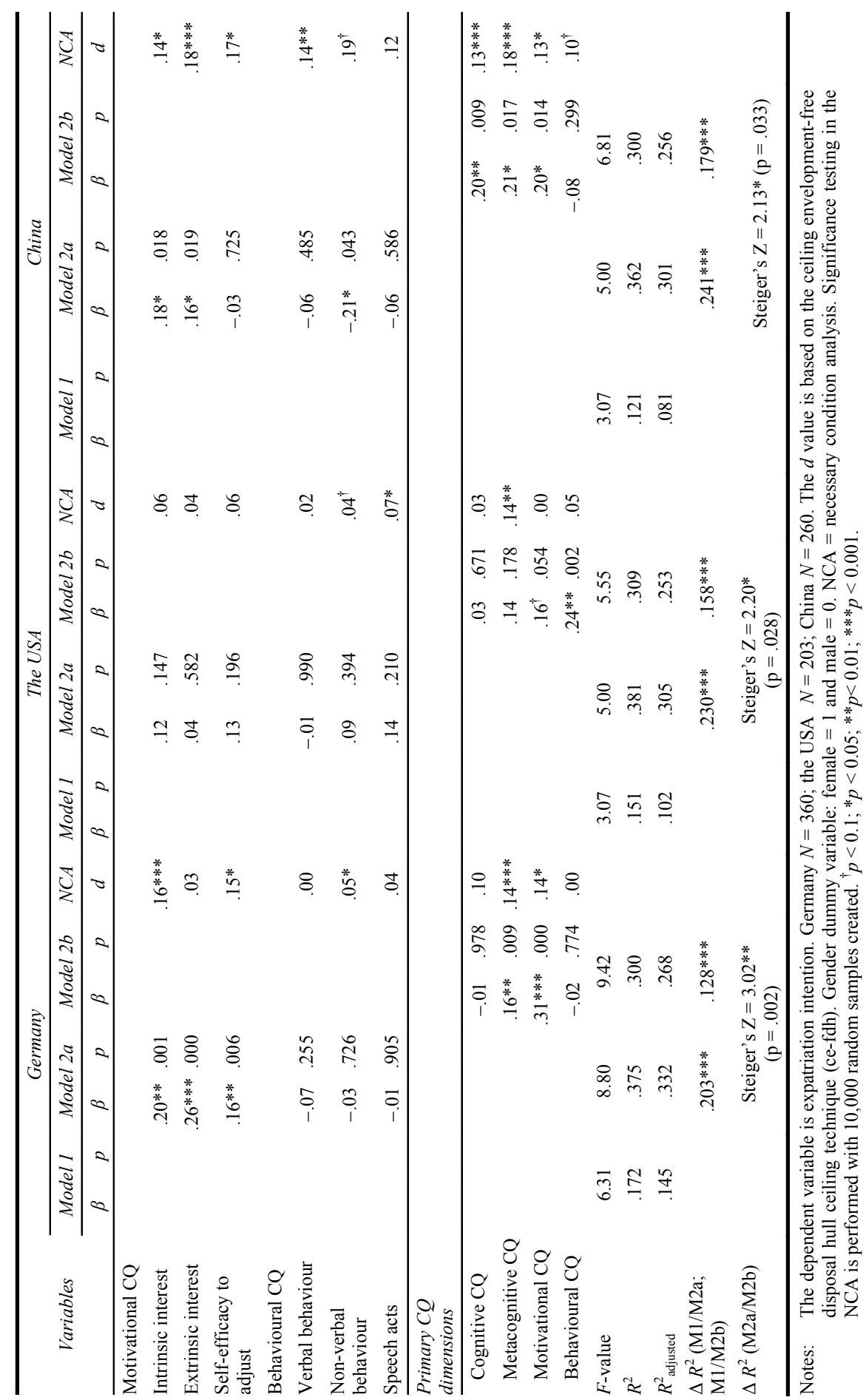


We conclude that Hypothesis 1 is partially confirmed for the German sample. Metacognitive CQ and motivational CQ are necessary conditions for EI and are positively associated with it. Hypothesis 2 is confirmed for the German sample. The expanded model of CQ is better able to explain EI than the original model of CQ.

\subsection{Results for the US sample}

In the following, we present the results for the US sample, summarised in the centre of Table 5. The control model has a moderate explanatory power for EI $\left(R^{2}=.15 ; R_{\text {adjusted }}^{2}=\right.$ .10). In Model 2, we add the 11 sub-dimensions of CQ. This significantly increases the explanatory power $\left(R^{2}=.38 ; R^{2}\right.$ adjusted $\left.=.31\right)$. Among the 11 sub-dimensions of $\mathrm{CQ}$, there is only one which significantly (and positively) influences EI: context-specific knowledge $(\beta=.25 ; p=.015)$. In Model $2 \mathrm{~b}$, we add the four primary dimensions as defined in the original CQS. This increases the amount of explained variance as compared to the control model significantly $\left(R^{2}=.31 ; R_{\text {adjusted }}^{2}=.25\right)$, yet the explanatory power is smaller if compared to the expanded CQ model. A Steiger's $Z$ test shows that the $R^{2}$-values between Model $2 \mathrm{a}$ and Model $2 \mathrm{~b}$ differ significantly, i.e. the expanded model (Model 2a) explains EI significantly better than Model 2b $(Z=2.20 ; p=.028)$. Among the four primary CQ dimensions, two are positive significant determinants of EI: motivational CQ $(\beta=.16 ; p=.054)$ and behavioural CQ $(\beta=.24 ; p=.002)$. From the $\mathrm{NCA}$, we identify one medium high and significant necessary condition effect size for the CQ sub-dimensions, namely for awareness $(d=.16 ; p=.000)$ of metacognitive CQ. Among the four original dimensions of the CQ model, metacognitive CQ $(d=.14$; $p=.008)$ is a medium high and significant necessary condition for EI.

We conclude that Hypothesis 1 is partially confirmed for the US sample. Metacognitive CQ is a necessary condition for EI and motivational CQ and behavioural CQ are positively associated with it. The expanded model of CQ is better able to explain EI than the original CQ model, supporting Hypothesis 2 for the US sample.

\subsection{Results for the Chinese sample}

The right side of Table 5 provides the results for the Chinese sample. The control model has rather limited explanatory power EI $\left(R^{2}=.12 ; R_{\text {adjusted }}^{2}=.08\right)$. Adding the 11 subdimensions of CQ (Model 2a) significantly increases the explanatory power $\left(R^{2}=.36\right.$; $R_{\text {adjusted }}^{2}=.30$ ). The sub-dimensions of CQ which significantly determine EI in a positive way are context-specific knowledge $(\beta=.21 ; p=.029)$, the meta-cognitive CQ subdimension checking $(\beta=.30 ; p=.004)$ and two motivational CQ sub-dimensions, namely intrinsic interest $(\beta=.18 ; p=.018)$ and extrinsic interest $(\beta=.16 ; p=.019)$. Finally, non-verbal behaviour is significantly and negatively associated with EI $(\beta=-.21 ; p=.043)$. When we add the four primary CQ dimensions (Model 2b) the amount of explained variance is significantly increased as compared to the control model as well $\left(R^{2}=.30 ; R_{\text {adjusted }}^{2}=.26\right)$, however, Model $2 \mathrm{~b}$ also shows less explanatory power against the expanded CQ model. The Steiger's $Z$ test for the difference in $R^{2}$-values shows that the expanded model (Model 2a) explains EI significantly better than Model $2 \mathrm{~b}$ $(Z=2.13 ; p=.033)$. Among the four primary $\mathrm{CQ}$ dimensions, three are positive significant determinants of EI: cognitive CQ $(\beta=.197 ; p=.009)$, metacognitive CQ $(\beta=.21 ; p=.017)$ and motivational CQ $(\beta=.20 ; p=.014)$. 
The NCA for the Chinese sample reveals numerous medium high necessary conditions for EI. Among the 11 sub-dimensions of CQ, almost all are medium high and significant necessary conditions for EI, except for the metacognitive sub-dimension of planning and speech acts as part of behavioural CQ. The highest effects are found for checking $(d=.18 ; p=.000)$, extrinsic interest $(d=.18 ; p=.001)$ and self-efficacy to adjust $(d=.17 ; p=.011)$ (we will not comment on the effect for non-verbal behaviour, $d=.19$, due to the issues of discriminant validity, here). Furthermore, also the four original dimensions of CQ are nearly all medium high and significant necessary conditions for EI: cognitive CQ $(d=.13 ; p=.000)$, metacognitive CQ $(d=.18 ; p=.000)$ and motivational CQ $(d=.13 ; p=.021)$.

We conclude that Hypothesis 1 is partially confirmed for the Chinese sample. Cognitive $\mathrm{CQ}$, metacognitive $\mathrm{CQ}$ and motivational $\mathrm{CQ}$ are significant necessary conditions for EI and are positively associated with it. Finally, in support of Hypothesis 2, the expanded CQ model is better able to explain EI than the original CQ model for the Chinese sample.

\section{Discussion}

\subsection{Implications for theory and research}

The present study tested the explanatory power of the expanded cultural intelligence scale (Van Dyne et al., 2012) and its 11 CQ sub-dimensions in predicting EI in comparison to the four primary CQ dimensions. Our study showed that the expanded version increased the explanatory power of the model in Germany, the USA and China, suggesting that the expanded scale is better able to explain variance in the intention to accept a foreign assignment (see Table 6 for an overview). The E-CQS is well aligned with the theoretical concepts that underlie the four primary CQ dimensions. It introduces items that were implicit in the definitions of the four original dimensions and have not been operationalised on the scale-level, for instance, the aspect of planning as a subdimension of metacognitive $\mathrm{CQ}$ and the inclusion of context-specific knowledge in cognitive CQ. For this reason, researchers interested in achieving higher explanatory power are therefore advised to consider the E-CQS. While our analyses showed partial measurement invariance of the scale and discriminant validity for almost all subdimensions except for the behavioural CQ sub-dimensions, we advise researchers to carefully evaluate theses aspects before analysing findings.

Table 6 Overview of findings

\begin{tabular}{|c|c|c|c|c|c|c|}
\hline & \multicolumn{2}{|c|}{ Germany } & \multicolumn{2}{|c|}{ The USA } & \multicolumn{2}{|c|}{ China } \\
\hline & $\begin{array}{l}\text { Necessary } \\
\text { condition }\end{array}$ & $\begin{array}{l}\text { Positive } \\
\text { driver }\end{array}$ & $\begin{array}{l}\text { Necessary } \\
\text { condition }\end{array}$ & $\begin{array}{l}\text { Positive } \\
\text { driver }\end{array}$ & $\begin{array}{l}\text { Necessary } \\
\text { condition }\end{array}$ & $\begin{array}{l}\text { Positive } \\
\text { driver }\end{array}$ \\
\hline \multicolumn{7}{|c|}{$\begin{array}{l}\text { Primary CQ dimensions } \\
\text { (CQS) }\end{array}$} \\
\hline Cognitive CQ & & & & & Yes & Yes \\
\hline Metacognitive CQ & Yes & Yes & Yes & & Yes & Yes \\
\hline Motivational CQ & Yes & Yes & & Yes & Yes & Yes \\
\hline Behavioural CQ & & & & Yes & & \\
\hline
\end{tabular}


Table 6 Overview of findings (continued)

\begin{tabular}{|c|c|c|c|c|c|c|}
\hline & \multicolumn{2}{|c|}{ Germany } & \multicolumn{2}{|c|}{ The USA } & \multicolumn{2}{|c|}{ China } \\
\hline & $\begin{array}{l}\text { Necessary } \\
\text { condition }\end{array}$ & $\begin{array}{l}\text { Positive } \\
\text { driver }\end{array}$ & $\begin{array}{l}\text { Necessary } \\
\text { condition }\end{array}$ & $\begin{array}{l}\text { Positive } \\
\text { driver }\end{array}$ & $\begin{array}{l}\text { Necessary } \\
\text { condition }\end{array}$ & $\begin{array}{l}\text { Positive } \\
\text { driver }\end{array}$ \\
\hline \multicolumn{7}{|l|}{$\begin{array}{l}\text { CQ sub-dimensions } \\
\text { (E-CQS) }\end{array}$} \\
\hline \multicolumn{7}{|l|}{ Cognitive CQ } \\
\hline Culture-general knowledge & & & & & Yes & \\
\hline Context-specific knowledge & & & & Yes & Yes & Yes \\
\hline \multicolumn{7}{|l|}{ Metacognitive CQ } \\
\hline \multicolumn{7}{|l|}{ Planning } \\
\hline Awareness & & & Yes & & Yes & \\
\hline Checking & Yes & & & & Yes & Yes \\
\hline \multicolumn{7}{|l|}{ Motivational CQ } \\
\hline Intrinsic interest & Yes & Yes & & & Yes & Yes \\
\hline Extrinsic interest & & Yes & & & Yes & Yes \\
\hline Self-efficacy to adjust & Yes & Yes & & & Yes & \\
\hline \multicolumn{7}{|l|}{ Behavioural CQ } \\
\hline Verbal behaviour & & & & & Yes & \\
\hline Non-verbal behaviour & & & & & Yes & \\
\hline \multicolumn{7}{|l|}{ Speech acts } \\
\hline \multicolumn{7}{|l|}{$\begin{array}{l}\text { Comparison of explanatory } \\
\text { power of E-CQS vs. CQS }\end{array}$} \\
\hline E-CQS $>$ CQS & Yes & & Yes & & Yes & \\
\hline
\end{tabular}

Our second contribution is with regard to the theoretical understanding of how CQ influences expatriate intention. We provide a set of arguments on mechanisms how the CQ sub-dimensions are associated with EI with reference to the TPB. We argue that general and specific knowledge are associated with EI as they imply a higher perceived behavioural control. The same line of argument goes for planning, awareness and the three sub-dimensions of behavioural CQ. For motivational CQ, we argue that intrinsic interest influences EI as it implies a more positive attitude towards the entrepreneurial endeaveour; self-efficacy influences EI as it implies a higher perceived behavioural control and extrinsic interest influences EI via a more positive attitude towards EI and a higher subjective norm. We encourage authors to discuss and further test these mechanisms proposed. Our empirical findings confirm and complement the knowledge about the relationship between CQ and EI that is outlined in a recent meta-analysis on the association between CQ and EI (Schlägel et al., 2017); CQ is positively associated with EI - in particular motivational and metacognitive CQ are important drivers. In line with previous research (Kim and Froese, 2012; Remhof et al., 2013; Schlägel and Sarstedt, 2016), we found that motivational CQ is the strongest determinant which also fits to theorising as the motivational CQ sub-dimensions imply higher facets on all classical TPB determinants of EI. Our study moroever shows that particularly intrinsic interest and self-efficacy to adjust are relevant when it comes to accepting international assignments; in Germany and in China these are even necessary conditions. Yet also extrinsic 
incentives can increase EI which is in line with the more general research on motivation (e.g. Haines et al., 2008). Our study further shows the added value of context-specific knowledge - one of the sub-dimensions of cognitive CQ - for explaining EI. In the American and Chinese context, a higher context-specific knowledge qualifies as a driver of EI; in China it is, moreover, a necessary condition for EI, i.e. there is no EI without knowledge in a managerial context among Chinese business students.

Our study also adds to the literature by being among the first to examine these necessary conditions of EI, i.e. without which EI will not exist (Dul, 2016a). For the original CQ scale, we found that cognitive CQ (in one country), metacognitive CQ (in all three countries) and motivational CQ (in two countries) are necessary conditions for EI; behavioural CQ in contrast is not. The relative unimportance of the latter aspect could be explained by the expectation that behavioural CQ becomes most effective during and not before an intercultural interaction (Schlägel et al., 2017); in this case, the focus has been on intentions, not interactions. This suggest that if potential expatriates do not possess these particular aspects of CQ, there will be no expatriation intention.

Going more in-depth and looking at the expanded scale, a surprising aspect, however, was the fact that planning (a sub-dimension of metacognitive CQ) was not a necessary condition nor even a significant driver of EI in any of the country settings. This is surprising because many authors have relied heavily on arguments related to planning when building hypotheses with regard to the association between metacognitive CQ and EI. In addition, planning is a relevant aspect of perceived behavioural control in the TPB. This is another example of the value added by using the E-CQS; the 11 sub-dimensions of CQ provide more information which helps shed light on the exact nature of the link between CQ and EI.

A final aspect to discuss is the fact that our study took place in three different countries (China, Germany and the USA) to test whether the structure and relevance of CQ sub-dimensions varied across a set of countries. Our findings revealed that CQ is a useful determinant when it comes to explaining EI in all three country contexts. Yet, they also revealed that different CQ sub-dimensions are relevant in different countries. This indicates the importance of differences in institutional environments which influence individuals' intentions and behaviour. While it is outside the scope of this paper to discuss these differences in detail, we would recommend that future research explores these differences further, including even more countries to establish the cross-cultural validity of CQ or including constructs for institutional environments in empirical models. Therewith, researchers might more systematically examine the potential boundary conditions related to differences in the formal and informal institutional environments.

\subsection{Managerial implications}

Cultural intelligence is more crucial than ever in today's globalised world. CQ has been found to be positively related to various work-related outcomes (Schlägel et al., 2017), and, for this reason, it is an important concept for international human resource (IHR) managers when selecting and training employees. Our study focused on potential expatriates and showed that their CQ is positively associated with their intention to accept an international assignment.

Considering the increasing importance of CQ in the intercultural workplace, it could be advocated that multinational firms include CQ when selecting their new employees. This knowledge - particularly on the sub-dimension intrinsic interest - could then also 
be used to create a global talent pool of potential expatriates. Collings and Isichei (2018) posit that establishing a talent pool with suitably skilled and qualified individuals who would like to work abroad should be a main concern for multinational firms. This would allow for a more strategic approach to assignee selection which is linked to organisational strategy and individual career plans. Furthermore, with the rise of the dual career couple, it becomes more difficult than ever to convince expatriates to accept an international assignment (Caligiuri and Bonache, 2016). For this reason, identifying individuals who would like to work abroad right at the start of their career can be an important strategy to widen the global talent pool and improve the selection process for international assignments.

Our study further revealed the importance of the other two motivational subdimensions, namely self-efficacy to adjust and extrinsic interest. IHR managers that have the objective to spark interest in an expatriate position may use these insights to tailor programs (e.g. mentoring programs, see also Schuster et al., 2017) and training for specific target groups. For example, multinational firms could offer workshops for highprofile students, interns and current employees on cultural intelligence and working in culturally diverse environments that would enhance an individuals' self-efficacy to adjust. Companies could also expose them to current or former expatriates who have had great experiences. In such a workshop, multinational firms could also highlight the extrinsic incentives for prospective expatriates and the support provided for expatriation and repatriation, enhancing both the extrinsic interest and the self-efficacy to adjust. Furthermore, multinational firms are advised to pay particular attention to the repatriation phase in order to make sure an international assignment is seen as beneficial for one's career. Collings and Isichei (2018) in their review of the literature found support for the view that even though the value of international experience from a developmental perspective is widely accepted, it is not clear that organisations actually integrate development opportunities into career paths for those identified as talent. This may also influence the willingness of potential expatriates to accept an international assignment. Multinational firms should also pay attention to the expatriate compensation package to make sure they offer the right extrinsic motivation to stimulate potential expatriates to accept the international assignment. However, since the cost of these compensation packages is a major organisational concern (Caligiuri and Bonache, 2016), it is important to remember that our study showed the particular relevance of intrinsic interest and selfefficacy to adjust, suggesting the importance of selection and training emphasis in these related areas.

\subsection{Limitations and future research directions}

Our findings should be interpreted in light of the limitations of this study. First, to be able to generalise findings future research should use a more diverse sample, not only in terms of countries but also in terms of respondents from different majors (e.g. engineering, chemistry, design or medical), different universities and different regions of a country (e.g. China's coastal cities and provinces vs. inner provinces). It could also be interesting to include employees in their early career.

Second, our cross-sectional research design limits our ability to draw conclusions about the causal relationship between CQ and EI. Future research should utilise longitudinal studies and quasi experimental designs to validate our findings. 
Third, the current study used a self-rated instrument to assess the CQ sub-dimensions. While prior research that utilised the CQS found high correlations between self-assessed and others-assessed CQ scores (Shannon and Begley, 2008), we echo previous calls (Ott and Michailova, 2018) for more research employing peer-assessment and/or observation of CQ to rule out any confounding influence of the research method.

A fourth limitation is that we were unable to include both the CQS and E-CQS in the same survey due to length constraints and potential respondent fatigue bias. Instead, we compared both scales based on the overlap in the items included in both measures. Future research should consider including both measures in the same survey, as well as examining the value of the two instruments in different contexts and for different outcomes. Furthermore, in the context of expatriate intention, we lack an understanding if those individuals with a higher EI, once they are an expatriate, more easily adjust to their new environment and show a higher job performance, compared to those expatriates with a lower prior intention. Such research would allow evaluating the practical usefulness of $\mathrm{CQ}$ and EI with respect to the overall expatriate selection decision.

Finally, our test of the discriminant validity for all three country samples showed that especially the sub-dimensions of behavioural CQ have limited discriminant validity against each other. In some of the countries, they also do not discriminate enough from items used to operationalise metacognitive CQ. While, inspection of correlations and VIF indicates no problems, our results for these dimensions should be interpreted in light of this limitation. Hence, we advise researchers to further explore the discriminant validity of the new items and to evaluate if these are actually measuring the different facets theoretically outlined; there is a further need to test and refine the instrument and measurement items.

Our study provides two further interesting avenues for future research. First, more research is needed into the path leading from EI to actual behaviour (i.e. accepting an expatriate position). In the present study, we examined the direct relation between CQ dimensions and EI, however, this process is likely to be a more complex sequential process that may also involve situational and contextual conditions that moderate this process. For example, in the formation of intentions, an individual's desire to conduct a specific behaviour seems to be an important mediator of the relation between attitudes and intention (Bagozzi and Kimmel, 1995; Perugini and Bagozzi, 2004). The desire to work as an expatriate may function as mediator in the relation between intrinsic and extrinsic interests and EI. Furthermore, our findings provide initial evidence for national context-bound differences in intrinsic and extrinsic motivational orientations. Recent conceptual studies suggest the different parts of the national context directly and indirectly influence individuals' intentions and behaviour through their more distal influence on attitudes, perceptions and ways of knowing and their moderating role in the relation between attitudes etc. and intentions and behaviours (Tsui et al., 2007; Taras et al., 2011). Future research should explore potential mediators and moderators along these lines to provide a more detailed explanation of the mechanisms and process that determine EI and the actual behavioural decision to take specified action (i.e. accept an expatriate position). Finally, a likewise interesting future research avenue is to enrich the focus on expatriation to repatriation outcomes (Breitenmoser and Bader, 2016; Breitenmoser et al., 2017) and how these are affected by CQ and its sub-dimensions.

A final avenue for future research is a stronger focus on a configurational perspective and set-theoretic approach. Echoing prior calls for such research by Van Dyne et al. (2012) we encourage future research to examine the combinations of CQ dimensions and, 
therewith, the different paths that lead to specific outcomes, such as EI. Previous studies as well as the present study have tested the additive predictive ability of individual CQ dimensions, neglecting the possibility that these dimensions can have different associations depending on the interaction with other variables, resulting in a comparable outcome. Researchers could utilise fuzzy-set qualitative comparative analysis (fsQCA) to explore such complex recipes of antecedent conditions that lead to EI. The present study is a first step towards a more configurational perspective as we identified necessary conditions for EI to emerge, representing a basic building block required for fsQCA (Dul, 2016a; Vis and Dul, 2016). These directions for future inquiry do not encompass the only research questions that scholars may pursue, but we believe that both have the potential to contribute to the rapid developments in the measurement, operationalisation and validation of CQ as well as the identification and empirical testing of new theories and hypotheses that contribute to a better understanding of the development of EI.

\section{References}

Ajzen, I. (1991) 'Theory of planned behavior', Behavior and Human Decision Processes, Vol. 50, pp.179-211.

Alon, I., Boulanger, M., Meyers, J. and Taras, V. (2016) 'The development and validation of the business cultural intelligence quotient', Cross Cultural \& Strategic Management, Vol. 23, pp.78-100.

Andresen, M. and Bergdolt, F. (2016) 'A systematic literature review on the definitions of global mindset and cultural intelligence - merging two different research streams', The International Journal of Human Resource Management, Vol. 28, pp.170-195.

Ang, S. and Van Dyne, L. (2008) 'Conceptualization of cultural intelligence: definition, distinctiveness, and nomological network', in Ang, S. and Van Dyne, L. (Eds): Handbook of Cultural Intelligence: Theory, Measurement, and Applications, M.S. Sharpe, New York.

Ang, S., Van Dyne, L. and Koh, C. (2006) 'Personality correlated of the four-factor model of cultural intelligence', Group \& Organization Management, Vol. 31, pp.100-123.

Ang, S., Van Dyne, L., Koh, C., Ng, K.Y., Templer, K.J., Tay, C. and Chandrasekar, N.A. (2007) 'Cultural intelligence: its measurement and effects on cultural judgment and decision making, cultural adaptation and task performance', Management and Organization Review, Vol. 3, pp.335-371

Bader, B., Berg, N. and Holtbrügge, D. (2015) 'Expatriate performance in terrorism-endangered countries: the role of family and organizational support', International Business Review, Vol. 24, pp.849-860.

Bagozzi, R.P. and Kimmel, S.K. (1995) 'A comparison of leading theories for the prediction of goal-directed behaviours', British Journal of Social Psychology, Vol. 34, pp.437-461.

Baluku, M.M., Löser, D., Otto, K. and Schummer, S.E. (2018) 'Career mobility in young professionals: how a protean career personality and attitude shapes international mobility and entrepreneurial intentions', Journal of Global Mobility, Vol. 6, pp.102-122.

Bandura, A. (1986) Social Foundations of Thought and Action: A Social Cognitive Theory, Prentice-Hall, Inc, Englewood Cliffs, NJ.

Bello, D., Leung, K., Radebaugh, L., Tung, R.L. and Van Witteloostuijn, A. (2009) 'From the editors: student samples in international business research', Journal of International Business Studies, Vol. 40, pp.361-364.

Breitenmoser, A. and Bader, B. (2016) 'Repatriation outcomes affecting corporate ROI: a critical review and future agenda', Management Review Quarterly, Vol. 66, pp.195-234. 
Breitenmoser, A., Bader, B. and Berg, N. (2017) 'Why does repatriate career success vary? An empirical investigation from both traditional and protean career perspectives', Human Resource Management, Vol. 57, pp.1049-1063.

Brislin, R.W. (1980) 'Translation and content analysis of oral and written material', in Triandis, H.C. and Berry, J.W. (Eds): Handbook of Cross-Cultural Psychology.

Caligiuri, P. and Bonache, J. (2016) 'Evolving and enduring challenges in global mobility', Journal of World Business, Vol. 51, pp.127-141.

Caligiuri, P.M. (2000) 'Selecting expatriates for personality characteristics: a moderating effect of personality on the relationship between host national contact and cross-cultural adjustment', MIR: Management International Review, Vol. 40, pp.61-80.

Cerasoli, C.P., Nicklin, J.M. and Ford, M.T. (2014) 'Intrinsic motivation and extrinsic incentives jointly predict performance: a 40-year meta-analysis', Psychological Bulletin, Vol. 140, pp. $980-1008$.

Chen, A.S-Y., Wu, I.H. and Bian, M-D. (2014) 'The moderating effects of active and agreeable conflict management styles on cultural intelligence and cross-cultural adjustment', International Journal of Cross Cultural Management, Vol. 14, pp.270-288.

Collings, D., Scullion, H. and Morley, M. (2007) 'Changing patterns of global staffing in the multinational enterprise: challenges to the conventional expatriate assignment and emerging alternatives', Journal of World Business, Vol. 42, pp.198-213.

Collings, D.G. and Isichei, M. (2018) 'The shifting boundaries of global staffing: integrating global talent management, alternative forms of international assignments and non-employees into the discussion', The International Journal of Human Resource Management, Vol. 29, pp.165-187.

Crowne, K.A. and Engle, R.L. (2016) 'Antecedents of cross-cultural adaptation stress in short-term international assignments', Organization Management Journal, Vol. 13, pp.32-47.

Donnellan, M.B., Oswald, F.L., Baird, B.M. and Lucas, R.E. (2006) 'The mini-IPIP scales: tinyyet-effective measures of the Big Five factors of personality', Psychological Assessment, Vol. 18, pp.192-203.

Dul, J. (2016a) 'Identifying single necessary conditions with NCA and fsQCA', Journal of Business Research, Vol. 69, pp.1516-1523.

Dul, J. (2016b) Necessary Condition Analysis, R Package Version 2.0 ed.

Dul, J. (2018) Necessary Condition Analysis (NCA) with R. R Package Version 3.0 ed.

Dul, J., Van Der Laan, E. and Kuik, R. (2018) 'A statistical significance test for necessary condition analysis', Organizational Research Methods, pp.1-11.

Earley, P.C. and Ang, S. (2003) Cultural Intelligence: Individual Interactions Across Cultures, Stanford University Press, Palo Alto, CA.

Engle, R.L., Schlägel, C., Dimitriadi, N., Tatoglu, E. and Ljubica, J. (2015) 'The intention to become an expatriate: a multinational application of the theory of planned behaviour', European Journal of International Management, Vol. 9, pp.108-137.

Fishbein, M. and Ajzen, I. (1975) Belief, Attitude, Intention, and Behavior: An Introduction to Theory and Research, Addison-Wesley, Reading, MA.

Ford, J.B. (2016) 'Cost vs credibility: the student sample trap in business research', European Business Review, Vol. 28, pp.652-656.

Froese, F. and Peltokorpi, V. (2013) 'Organizational expatriates and self-initiated expatriates: differences in cross-cultural adjustment and job satisfaction', The International Journal of Human Resource Management, Vol. 24, pp.1953-1967.

Gelfand, M.J., Imai, L. and Fehr, R. (2008) 'Thinking intelligently about cultural intelligence: the road ahead', in Ang, S. and Van Dyne, L. (Eds): Handbook of Cultural Intelligence: Theory, Measurement, and Applications, M.E. Sharpe, New York.

Grubb, B.A. (2015) A Study of Authentic Leadership and Cultural Intelligence in Higher Education Academic Leaders, Dissertation, Indiana Wesleyan University. 
Haines, V.Y., Saba, T. and Choquette, E. (2008) 'Intrinsic motivation for an international assignment', International Journal of Manpower, Vol. 29, pp.443-461.

Hair, J.F., Hult, G.T.M., Ringle, C.M., Sarstedt, M., Richter, N.F. and Hauff, S. (2017) Partial Least Squares Strukturgleichungsmodellierung (PLS-SEM): Eine anwendungsorientierte Einführung, Vahlen, München.

Harzing, A-W., Reiche, B.S. and Pudelko, M. (2013) 'Challenges in international survey research: a review with illustrations and suggested solutions for best practice', European Journal of International Management, Vol. 7, pp.112-134.

Hauff, S., Richter, N.F. and Tressin, T. (2015) 'Situational job characteristics and job satisfaction: the moderating role of culture', International Business Review, Vol. 24, pp.710-723.

Henseler, J., Ringle, C.M. and Sarstedt, M. (2016) 'Testing measurement invariance of composites using partial least squares', International Marketing Review, Vol. 33, pp.405-431.

Hoerger, M. (2013) $Z_{H}$ : an updated version of Steiger's $Z$ and web-based calculator for testing the statistical significance of the difference between dependent correlations. Available online at: http://www.psychmike.com/dependent_correlations.php

Huff, K.C. (2013) 'Language, cultural intelligence, and expatriate success', Management Research Review, Vol. 36, pp.596-612.

Isichei, M. (2017) Cultural Identification and Global Mobility: Exploring the Impact of Monoculturalism and Biculturalism on Willingness to Work Internationally and Effectiveness while on International Assignments, Dissertation, Dublin City University.

Kanfer, R. (2012) 'Work motivation: theory, practice, and future directions', in Kozlowski, S.W. (Ed): The Oxford Handbook of Organizational Psychology, Blackwell, Oxford, UK.

Kim, J. and Froese, F.J. (2012) 'Expatriation willingness in Asia: the importance of host-country characteristics and employees' role commitments', The International Journal of Human Resource Management, Vol. 23, pp.3414-3433.

Kolb, D. (1984) Experiential Learning as the Science of Learning and Development, Prentice Hall, Englewood Cliffs, NJ.

Leung, K. (2008) 'Methods and measurements in cross-cultural management', in Smith, P.B., Peterson, M.F. and Thomas, D.C. (Eds): The Handbook of Cross-Cultural Management Research, Sage, Los Angeles, CA

Matsumoto, D. and Hwang, H.C. (2013) 'Assessing cross-cultural competence: a review of available tests', Journal of Cross-Cultural Psychology, Vol. 44, pp.849-873.

McComas, A.B. (2014) The Relationship of a Leader's Cultural Intelligence to Organizational Citizenship Behaviors in a Multicultural Work Group, Dissertation, Indiana Wesleyan University.

Mcevoy, G.M. and Buller, P.F. (2013) 'Research for practice: the management of expatriates', Thunderbird International Business Review, Vol. 55, pp.213-226.

Mol, S.T., Born, M.P., Willemsen, M.E., Van Der Molen, H.T. and Derous, E. (2009) 'When selection ratios are high: predicting the expatriation willingness of prospective domestic entrylevel job applicants', Human Performance, Vol. 22, pp.1-22.

Mor, S. (2013) Cultural Metacognitive Processes: Psychological Mechanisms Promoting Intercultural Effectiveness, Dissertation, Columbia University.

Mor, S., Morris, M.W. and Joh, J. (2013) 'Identifying and training adaptive cross-cultural management skills: the crucial role of cultural metacognition', Academy of Management Learning \& Education, Vol. 12, pp.453-475.

Ott, D.L. and Michailova, S. (2018) 'Cultural intelligence: a review and new research avenues', International Journal of Management Reviews, Vol. 20, pp.99-119.

Perugini, M. and Bagozzi, R.P. (2004) 'The distinction between desires and intentions', European Journal of Social Psychology, Vol. 34, pp.69-84.

Peterson, R.A. and Merunka, D.R. (2014) 'Convenience samples of college students and research reproducibility', Journal of Business Research, Vol. 67, pp.1035-1041. 
Philippart, N. (2014) Global e-mentoring: Overcoming Virtual Distance for an Effective Mentoring Relationship, Dissertation, Wayne State University.

Pinto, L., Bader, B. and Schuster, T. (2017) 'Dangerous settings and risky international assignments', Journal of Global Mobility: The Home of Expatriate Research, Vol. 5, pp.342-347.

Podsakoff, P.M. and Organ, D.W. (1986) 'Self reports in organizational research: problems and prospects', Journal of Management, Vol. 12, pp.531-544.

Presbitero, A. and Quita, C. (2017) 'Expatriate career intentions: links to career adaptability and cultural intelligence', Journal of Vocational Behavior, Vol. 98, pp.118-126.

Racicot, B.M. and Ferry, D.L. (2016) 'The impact of motivational and metacognitive cultural intelligence on the study abroad experience', Journal of Educational Issues, Vol. 2, pp.115-129.

Remhof, S., Gunkel, M. and Schlägel, C. (2013) "Working in the "global village": the influence of cultural intelligence on the intention to work abroad', German Journal of Human Resource Management, Vol. 27, pp.224-250.

Richter, N.F., Sinkovics, R.R., Ringle, C.M. and Schlägel, C. (2016) 'A critical look at the use of SEM in International Business research', International Marketing Review, Vol. 33, pp.376-404.

Ringle, C.M., Wende, S. and Becker, J-M. (2015) SmartPLS 3, SmartPLS, Bönningstedt. Available online at: http://www.smartpls.com

Rockstuhl, T., Ang, S., Ng, K-Y., Lievens, F. and Van Dyne, L. (2015) 'Putting judging situations into situational judgment tests: evidence from intercultural multimedia SJTs', Journal of Applied Psychology, Vol. 100, pp.464-480.

Rockstuhl, T. and Van Dyne, L. (2018) 'A bi-factor theory of the four-factor model of cultural intelligence: meta-analysis and theoretical extensions', Organizational Behavior and Human Decision Processes, Vol. 148, pp.124-144.

Ronen, S. and Shenkar, O. (2013) 'Mapping world cultures: cluster formation, sources and implications', Journal of International Business Studies, Vol. 44, pp.867-897.

Ryan, R.M. and Deci, E.L. (2000) 'Self-determination theory and the facilitation of intrinsic motivation, social development, and well-being', American Psychologist, Vol. 55, pp.68-78.

Savickas, M.L. and Porfeli, E.J. (2012) 'Career Adapt-Abilities Scale: construction, reliability, and measurement equivalence across 13 countries', Journal of Vocational Behavior, Vol. 80, pp.661-673.

Schlägel, C., Richter, N. and Taras, V. (2017) 'Cultural intelligence and work-related outcomes: a meta-analytic review', Academy of Management Proceedings, 2017, Academy of Management Briarcliff Manor, NY 10510, 14152.

Schlägel, C. and Sarstedt, M. (2016) 'Assessing the measurement invariance of the fourdimensional cultural intelligence scale across countries: a composite model approach', European Management Journal, Vol. 34, pp.633-649.

Schuster, T., Ambrosius, J. and Bader, B. (2017) 'Mentoring in international assignments: a personality traits perspective', Employee Relations, Vol. 39, pp.1100-1130.

Shannon, L.M. and Begley, T.M. (2008) 'Antecedents of the four-factor model of cultural intelligence', in Ang, S. and Van Dyne, L. (Eds): Handbook of cultural Intelligence: Theory, Measurement, and Applications, M.E. Sharpe, New York.

Steiger, J.H. (1980) 'Tests for comparing elements of a correlation matrix', Psychological Bulletin, Vol. 87, pp.245-251.

Sternberg, R.J. (1999) 'The theory of successful intelligence', Review of General Psychology, Vol. 3, pp.292-316.

Stoermer, S., Davies, S.E., Bahrisch, O. and Portniagin, F. (2017) 'For sensation's sake: differences in female and male expatriates' relocation willingness to dangerous countries based on sensation seeking', Journal of Global Mobility, Vol. 5, pp.374-390. 
Taras, V., Steel, P. and Kirkman, B.L. (2011) 'Three decades of research on national culture in the workplace: do the differences still make a difference', Organizational Dynamics, Vol. 40, pp.189-198.

Templer, K.J., Tay, C. and Chandrasekar, N.A. (2006) 'Motivational cultural intelligence, realistic job preview, realistic living conditions preview, and cross-cultural adjustment', Group \& Organization Management, Vol. 31, pp.154-173.

Tharenou, P. (2003) 'The initial development of receptivity to working abroad: self-initiated international work opportunities in young graduate employees', Journal of Occupational and Organizational Psychology, Vol. 76, pp.489-515.

Thomas, D.C., Liao, Y., Aycan, Z., Cerdin, J-L., Pekerti, A.A., Ravlin, E.C., Stahl, G.K., Lazarova, M.B., Fock, H. and Arli, D. (2015) 'Cultural intelligence: a theory-based, short form measure', Journal of International Business Studies, Vol. 46, pp.1099-1118.

Tsui, A.S., Nifadkar, S.S. and Ou, A.Y. (2007) 'Cross-national, cross-cultural organizational behavior research: advances, gaps, and recommendations', Journal of Management, Vol. 33, pp.426-478

Van Dyne, L., Ang, S. and Koh, C. (2008) 'Development and validation of the CQS: the cultural intelligence scale', in Ang, S. and Van Dyne, L.V. (Eds): Handbook of Cultural Intelligence: Theory, Measurement and Applications, M.E. Sharpe, New York.

Van Dyne, L., Ang, S., Ng, K.Y., Rockstuhl, T., Tan, M.L. and Koh, C. (2012) 'Sub-dimensions of the four factor model of cultural intelligence: expanding the conceptualization and measurement of cultural intelligence', Social and Personality Psychology Compass, Vol. 6, pp.295-313.

Vis, B. and Dul, J. (2016) 'Analyzing relationships of necessity not just in kind but also in degree: complementing fsQCA with NCA', Sociological Methods \& Research, pp.1-28.

Yurtkoru, S.E., Dauda, E. and Sekarawisut, W. (2017) 'The theory of planned behavior augmented with cognitive cultural intelligence and work-related perceptions in predicting intention to work abroad', Journal of Advanced Management Science, Vol. 5, pp.176-181.

\section{Note}

1 A Steiger's $Z$ test is used to test whether two regression models have a significantly different explanatory power. The advantage of the test is that it can be used in the context of non-nested models; our Model 2 is not nested in Model 3, or in other words, Model 3 does not contain all independent variables of Model 2 plus further, but a different set of independent variables. 


\section{Appendices}

Table A1 Descriptive statistics and correlation coefficients for the German sample

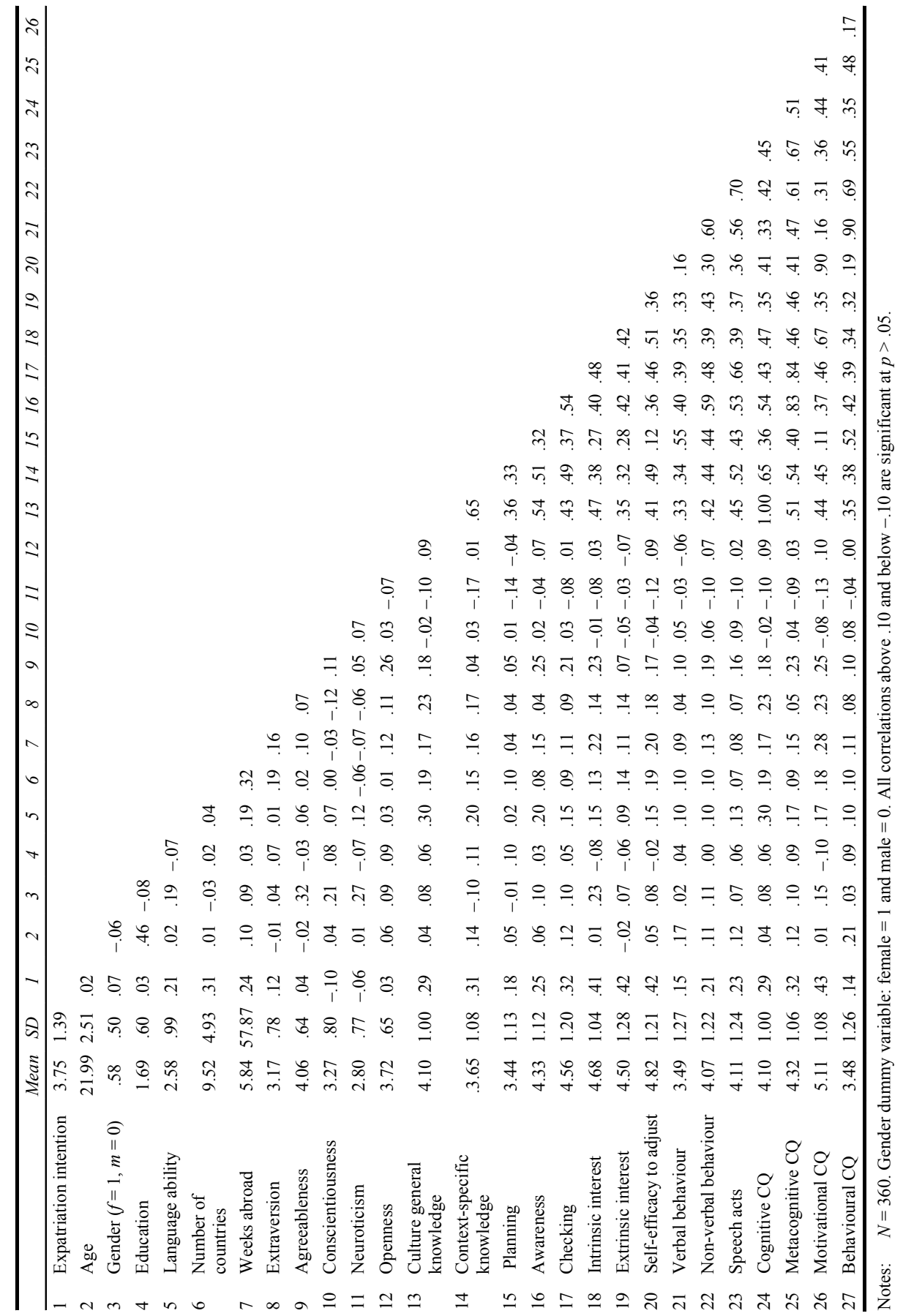


Table A2 Descriptive statistics and correlation coefficients for the US sample

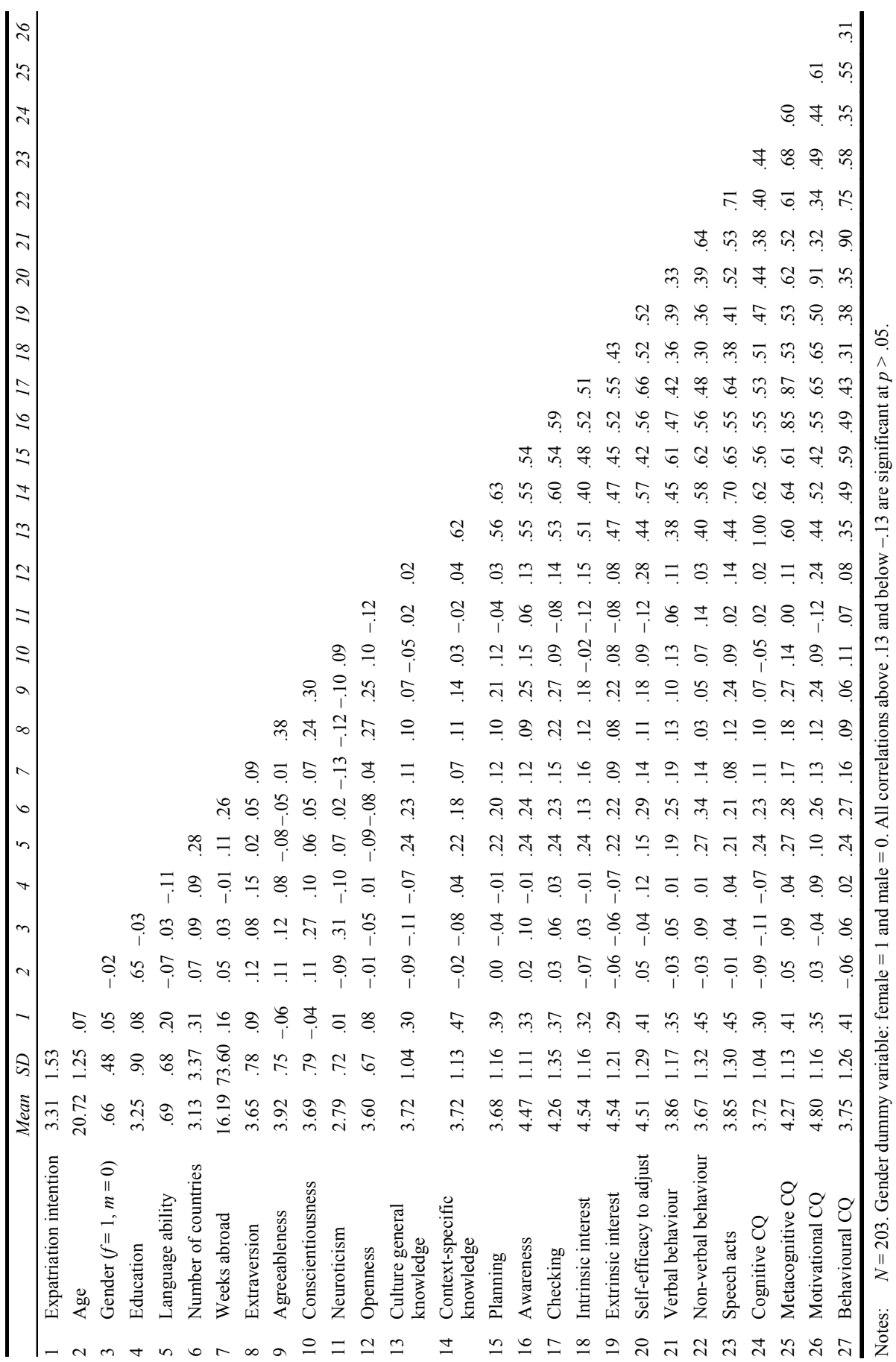


Table A3 Descriptive statistics and correlation coefficients for the Chinese sample

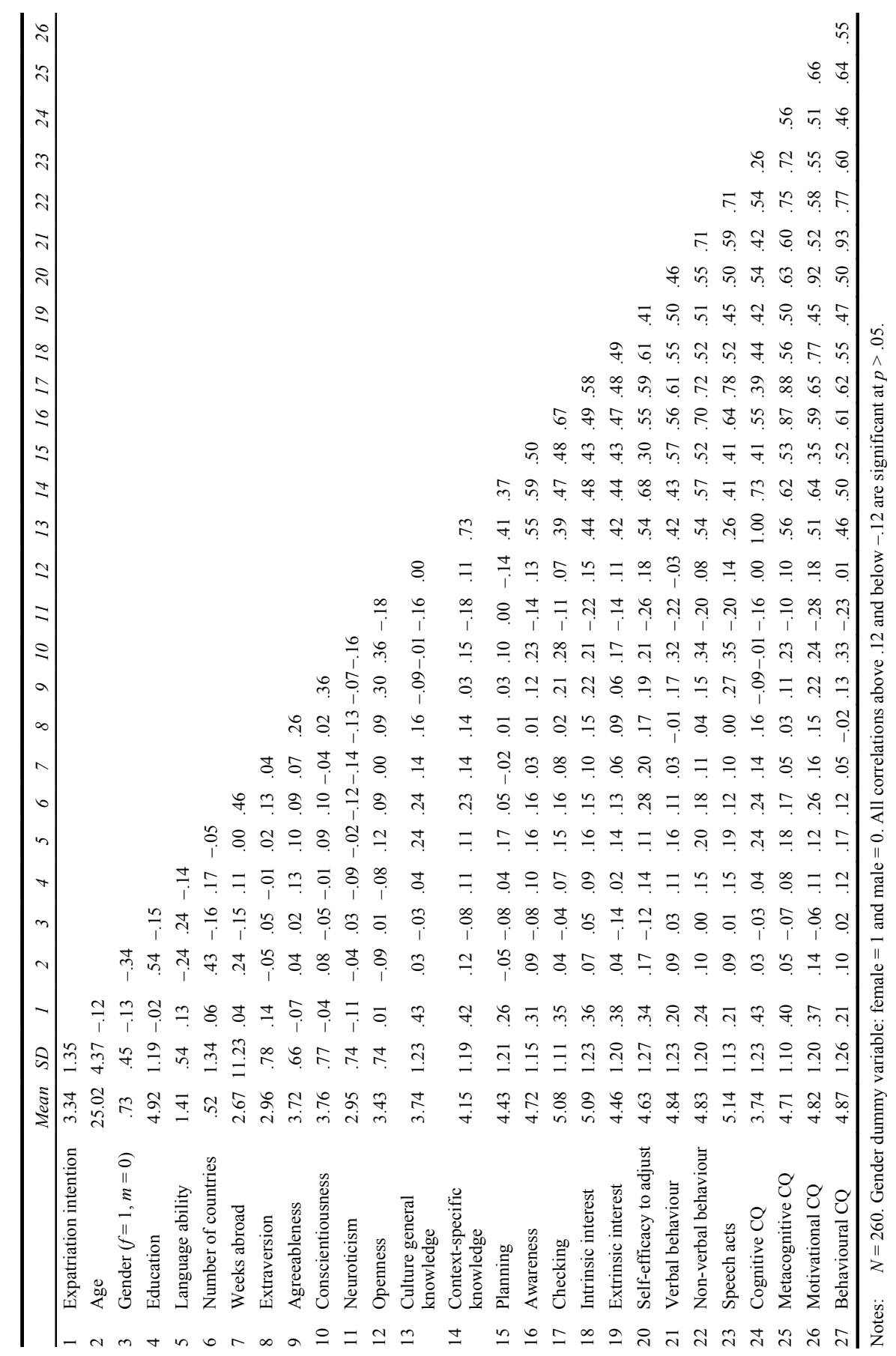

